\title{
Minimally invasive glaucoma surgery: current status and future prospects
}

\author{
This article was published in the following Dove Press journal: \\ Clinical Ophthalmology \\ 28 January 2016 \\ Number of times this article has been viewed
}

\author{
Grace M Richter ${ }^{1,2}$ \\ Anne L Coleman' \\ 'UCLA Stein Eye Institute, \\ Department of Ophthalmology, \\ University of California, Los Angeles, \\ CA, USA; ${ }^{2}$ USC Eye Institute, \\ Department of Ophthalmology, \\ Keck School of Medicine of University \\ of Southern California, Los Angeles, \\ CA, USA
}

\begin{abstract}
Minimally invasive glaucoma surgery aims to provide a medication-sparing, conjunctival-sparing, ab interno approach to intraocular pressure reduction for patients with mild-to-moderate glaucoma that is safer than traditional incisional glaucoma surgery. The current approaches include: increasing trabecular outflow (Trabectome, iStent, Hydrus stent, gonioscopy-assisted transluminal trabeculotomy, excimer laser trabeculotomy); suprachoroidal shunts (Cypass micro-stent); reducing aqueous production (endocyclophotocoagulation); and subconjunctival filtration (XEN gel stent). The data on each surgical procedure for each of these approaches are reviewed in this article, patient selection pearls learned to date are discussed, and expectations for the future are examined.
\end{abstract}

Keywords: MIGS, microincisional glaucoma surgery, trabecular stent, Schlemm's canal, suprachoroidal shunt, ab interno

\section{Introduction}

Glaucoma is the leading cause of irreversible blindness worldwide. It is estimated that 64.3 million people, or $3.54 \%$ of the world's population, have glaucoma. ${ }^{1}$ Currently, the only proven treatment for glaucoma is to lower intraocular pressure (IOP) with the goal of preventing additional glaucomatous optic nerve damage. ${ }^{2}$ While many patients can be controlled with medications, patient adherence and ocular toxicity are major issues in the developed world, and lifetime costs of and accessibility to medications are issues in developing regions. ${ }^{3}$ Selective laser trabeculoplasty (SLT) is a noninvasive, medication-sparing therapy that lowers IOP by $\geq 20 \%$ below baseline pressure in $66.7 \%-75 \%$ at 6 months but $11.1 \%-31 \%$ at 5 years; its effectiveness generally decreases over time. ${ }^{4,5}$ In the remainder of cases, traditional incisional surgery, which includes trabeculectomy or aqueous tube shunts, is performed. While generally the most effective IOP-lowering treatments available, they are accompanied by less than ideal risk profiles. ${ }^{6}$ The average IOP 5 years after surgery in the TVT study was $14.4 \mathrm{mmHg}$ in the tube group and $12.6 \mathrm{mmHg}$ in the trabeculectomy group. ${ }^{7}$ The rate of reoperation was $9 \%$ in the tube group and $29 \%$ in the trabeculectomy group at 5 years. ${ }^{7}$ These incisional surgeries can result in failure due to scarring, decreased quality of life due to bleb-related foreign body sensation, induced astigmatism, and secondary cataracts. ${ }^{6}$ Tube and trabeculectomy patients have similar rates of vision-threatening complications such as blebitis, endophthalmitis, or choroidal hemorrhage. ${ }^{6}$

Minimally invasive (or microincisional) glaucoma surgery (MIGS) aims to provide a safer, less invasive means of reducing IOP than traditional surgery, with the goal of reducing dependency on topical medications. Often, MIGS can be combined with cataract surgery via phacoemulsification, and in fact, in most clinical trials, they have been combined with cataract surgery. To date, the available MIGS procedures
UCLA Stein Eye Institute, 100 Stein Plaza,

Los Angeles, CA 90095, USA

Email coleman@jsei.ucla.edu 
offer more modest results than traditional glaucoma surgery, but with the benefit of a safer risk profile. Thus, these procedures are currently targeted at patients with mild-tomoderate glaucoma. In this review, we define MIGS as any glaucoma procedure avoiding conjunctival dissection and thus approached via an ab interno incision (clear corneal wound). The authors feel that because, at the present time, MIGS procedures as a whole are indicated for patients with glaucoma less severe than that requiring traditional incisional surgery, MIGS procedures by definition should preserve the conjunctiva from surgical dissection.

The four main approaches of IOP reduction by MIGS include increasing trabecular outflow by bypassing the juxtacanalicular trabecular meshwork (TM), increasing uveoscleral outflow via suprachoroidal pathways, reducing aqueous production from the ciliary body, or creating a subconjunctival drainage pathway. The benefits and limitations of each of the MIGS procedure classes, a review of the evidence of each MIGS procedure, and adverse events with clinical considerations will be discussed. A summary of the evidence for each device is provided in Table 1. Following this, the potential benefits of MIGS to glaucoma management on the whole, as well as limitations of current data, will be discussed.

\section{Increasing trabecular flow}

There are currently five MIGS procedures available in humans that target the juxtacanalicular portion of the TM: Trabectome, iStent, Hydrus, gonioscopy-assisted transluminal trabeculotomy (GATT), and excimer laser trabeculotomy (ELT). These procedures are based on the concept that the juxtacanalicular system is the site of greatest resistance of aqueous outflow in most open-angle glaucoma (OAG) patients. ${ }^{8}$ Like trabeculotomy for infants with congenital glaucoma, which removes the juxtacanalicular TM and inner wall of Schlemm's canal, these procedures aim to remove or bypass the inner wall of Schlemm's canal and allow aqueous more direct access from the anterior chamber to the collector channels at the outer aspect of Schlemm's canal. One challenge of this approach, especially with a focal stent, lies in the fact that the stent may not necessarily be positioned near one of the 25-30 collector channels in each eye. Additionally, some collector channels may be more active than others. Sometimes, the success of the procedure can also be limited by postoperative scarring at the site of Schlemm's canal. Finally, these procedures reduce juxtacanalicular resistance but do not reduce more distal sites of outflow resistance, such as elevated episcleral venous pressure, which may be more important in some patients. The postoperative IOP outcome after these procedures will not fall below episcleral venous pressure. As a general rule of thumb, these procedures should generally not be considered in patients with scleral buckles, disorders such as Grave's disease or Sturge-Weber syndrome, or patients with IOP targets below episcleral venous pressure.

\section{Trabectome}

The Trabectome system (Neomedix, Tustin, CA, USA) performs a trabeculotomy via an internal approach (Figure 1). It has been approved by the US Food and Drug Administration (FDA) since 2004 and has the Conformité Européenne (CE) mark of approval in Europe. Under the guidance of intraoperative gonioscopy, a disposable 19.5-gauge handpiece with an insulated footplate containing electrocautery, irrigation, and aspiration functions is inserted into the anterior chamber and then through the TM into Schlemm's canal. The device moves along the TM, removing both a strip of TM and the inner wall of Schlemm's canal. Thus a pathway for aqueous outflow from the anterior chamber directly into the collector channels is created. Usually $60^{\circ}-120^{\circ}$ of the nasal angle is treated..$^{9,10}$ A histopathologic study of treated corneoscleral rims have shown that the treated areas provide separation of the TM ends so that aqueous can have direct access to the collector channels. Additionally, there is no evidence of thermal damage to the surrounding tissues. ${ }^{11}$

\section{Efficacy}

There have been several studies on the Trabectome, however, all have been prospective noncomparative or retrospective case series on OAG patients. No randomized controlled trials have been performed to date.

\section{Trabectome alone}

In 2005, Minckler et al described the first cohort, followed for 13 months, of $37 \mathrm{OAG}$ patients to undergo Trabectome alone, and this cohort was expanded to 101 patients with a 30-month follow-up period in the report published in 2006. Results of these studies showed a reduction in IOP from $28.2 \pm 4.4 \mathrm{mmHg}$ to $16.3 \pm 2.0 \mathrm{mmHg}$ at 1 year and from $27.6 \pm 7.2 \mathrm{mmHg}$ to $15.2 \pm 2.4 \mathrm{mmHg}$ at 2 years, respectively, for each study. ${ }^{9,12}$ In 2013, Maeda et al ${ }^{13}$ reported results from Japan on 80 eyes of 69 patients undergoing Trabectome alone. He reported that mean IOP was reduced from 26.6 $\pm 8.1 \mathrm{mmHg}$ to $17.4 \pm 3.4 \mathrm{mmHg}$ at 6 months, and medications were reduced from $4.0 \pm 1.4$ to $2.3 \pm 1.2$. 


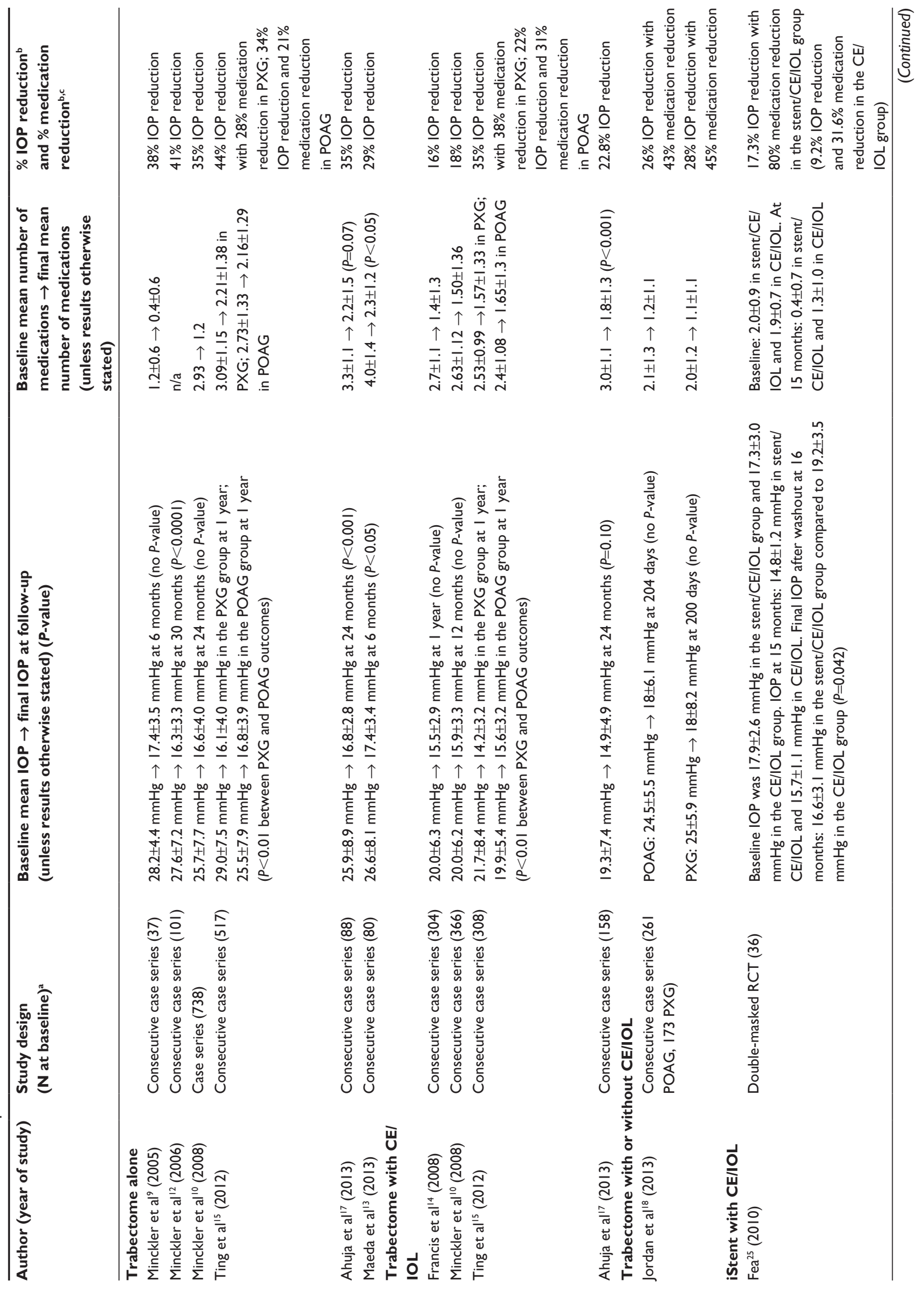




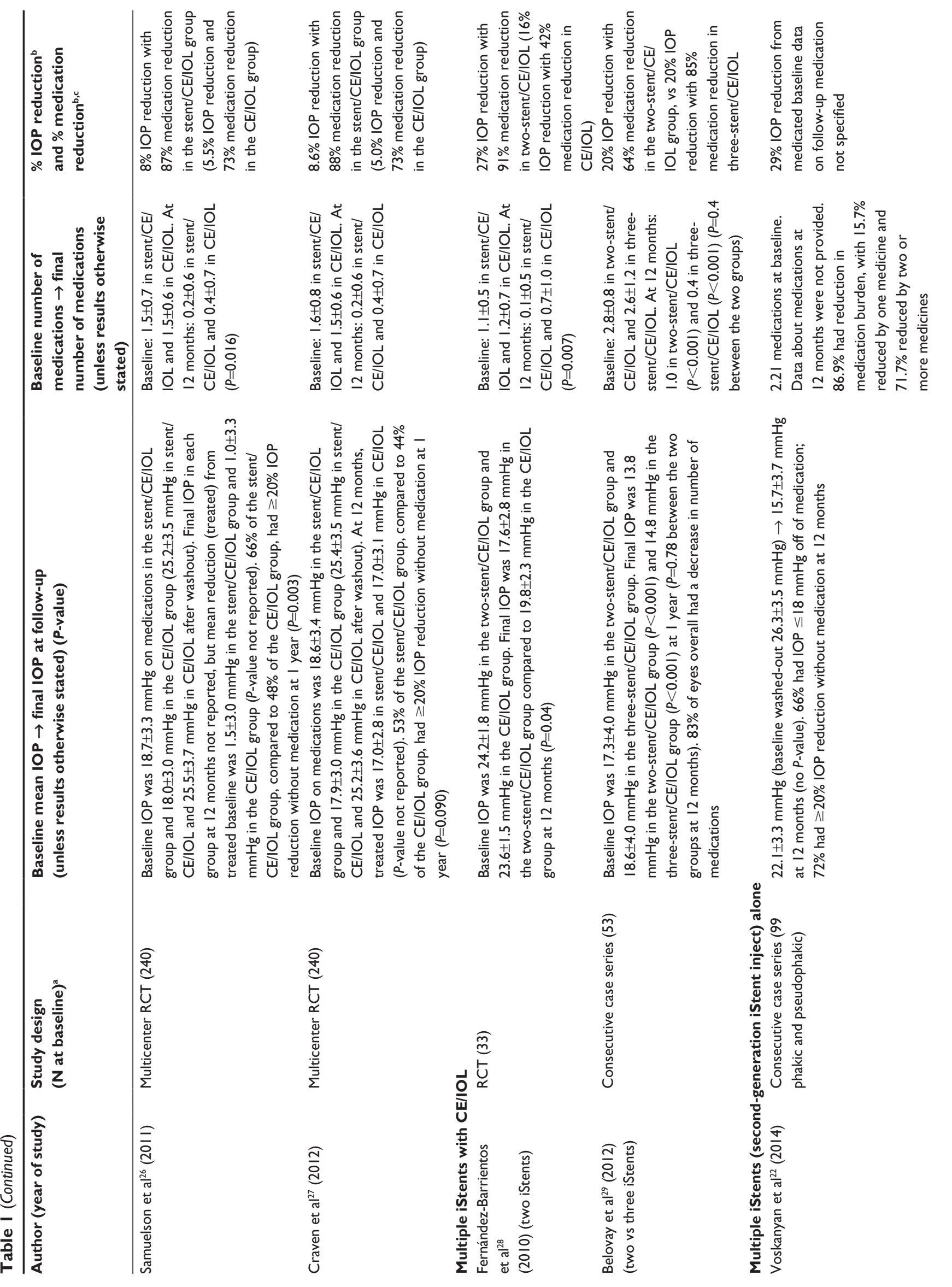



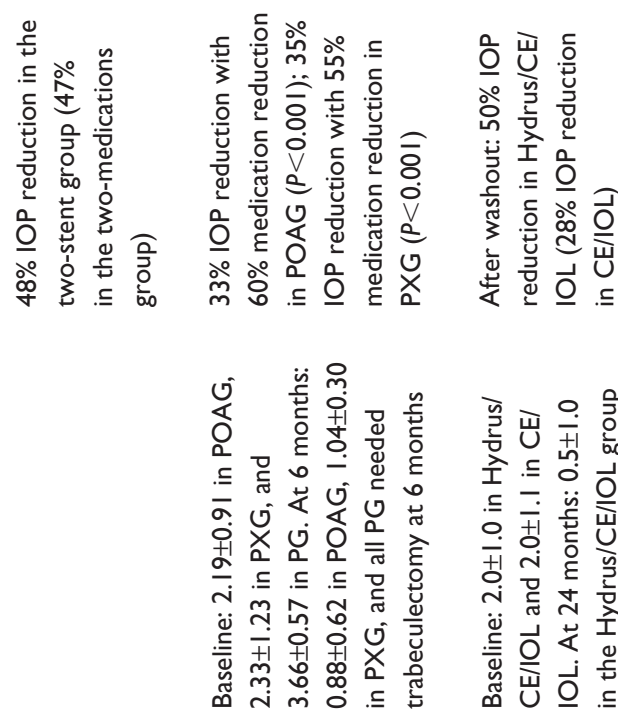

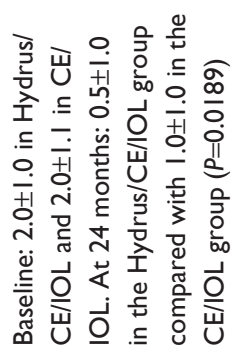

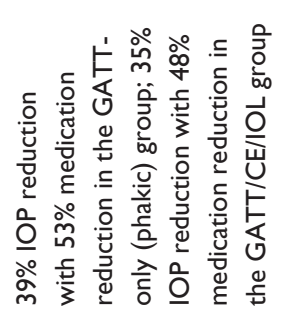

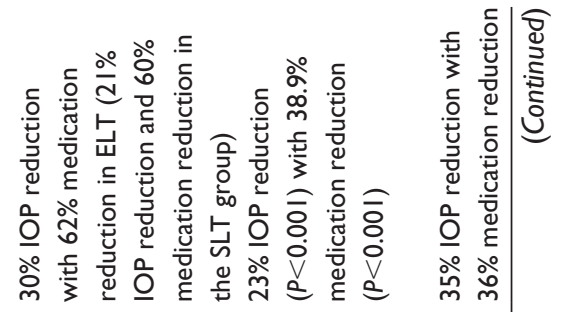

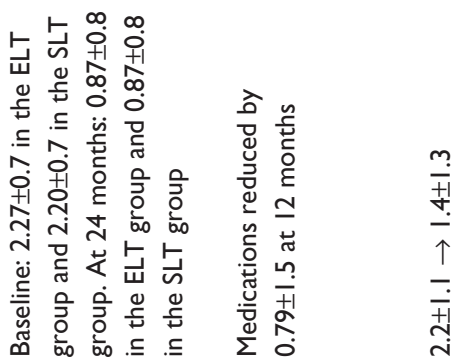
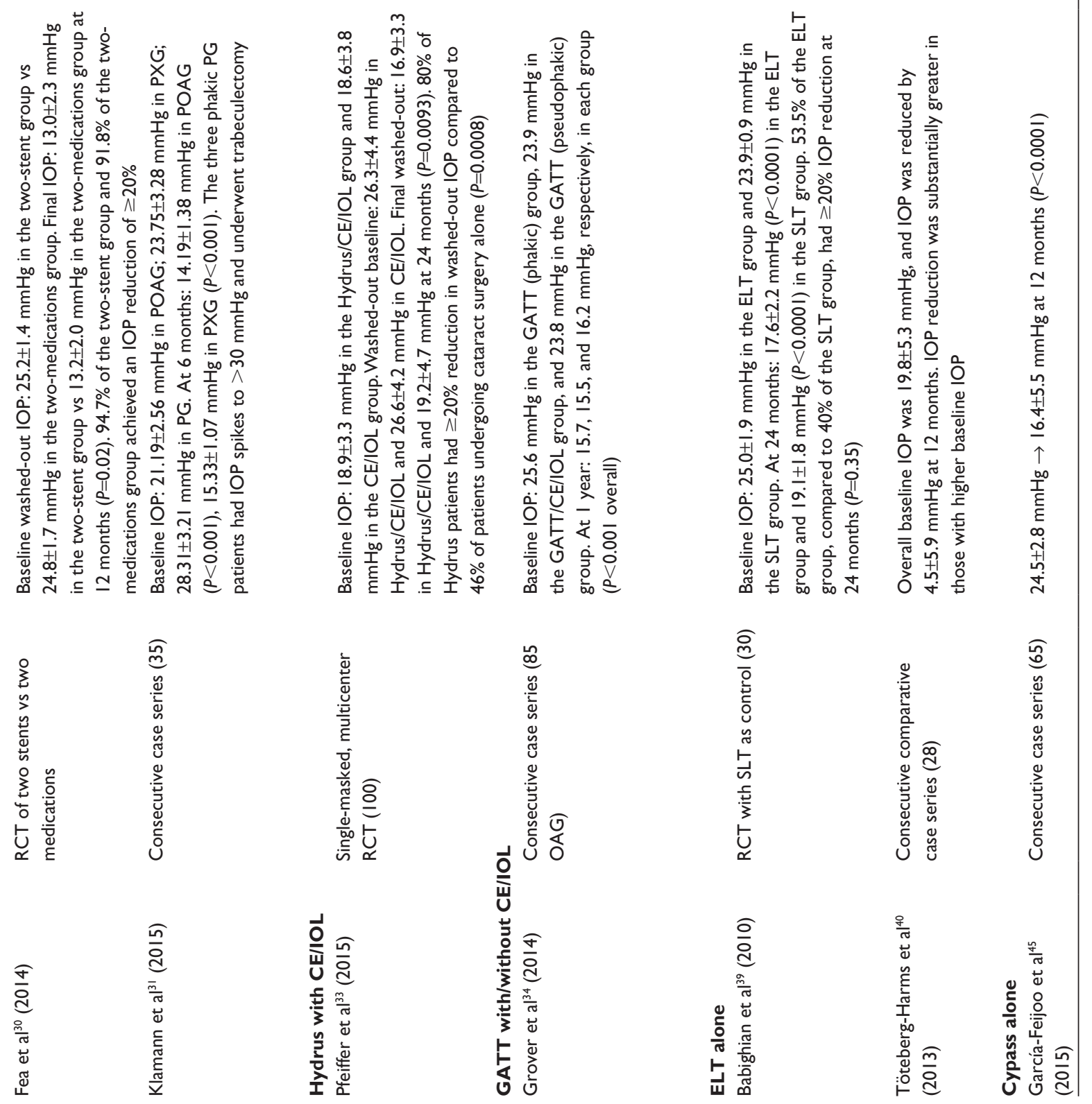


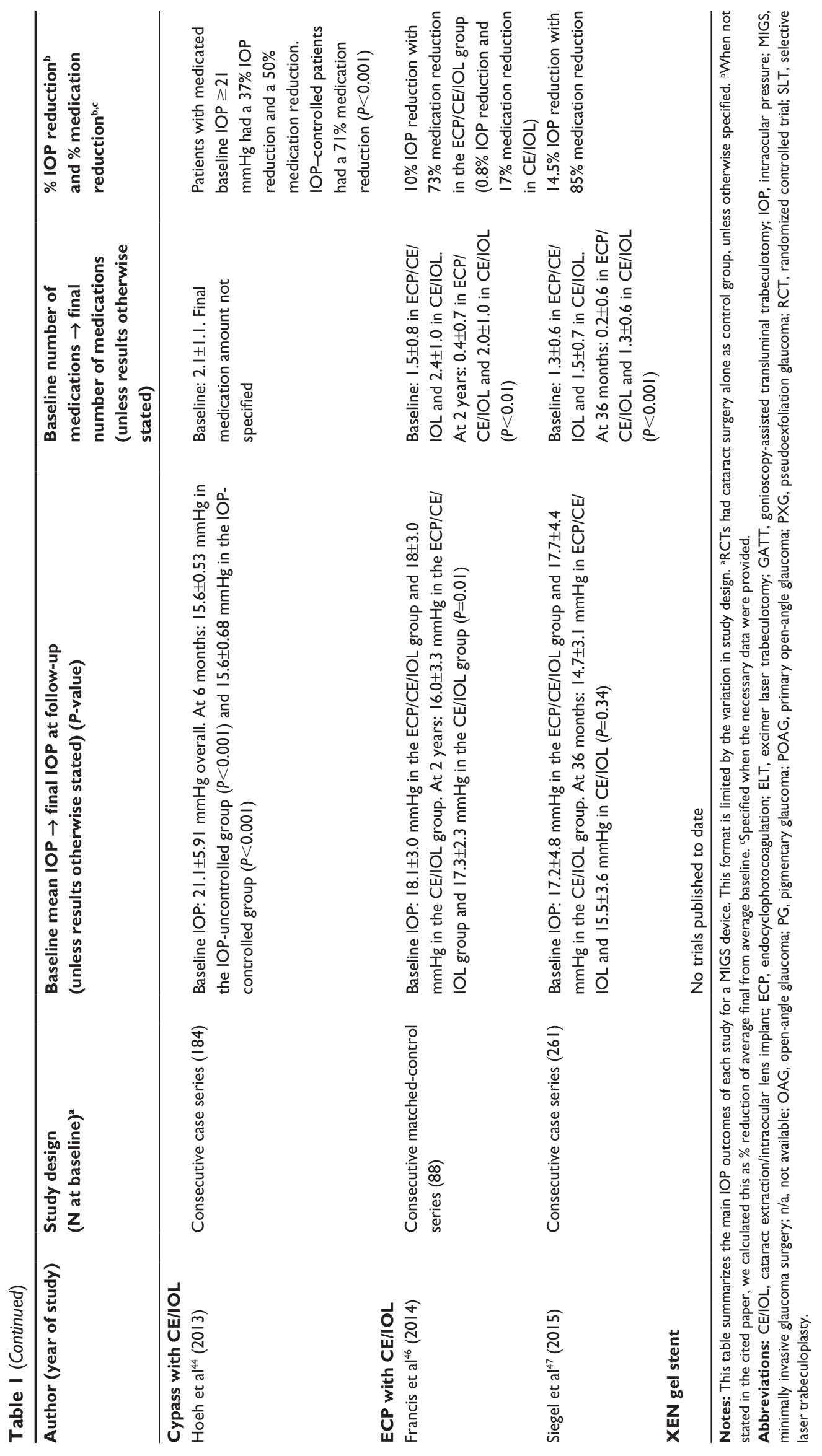




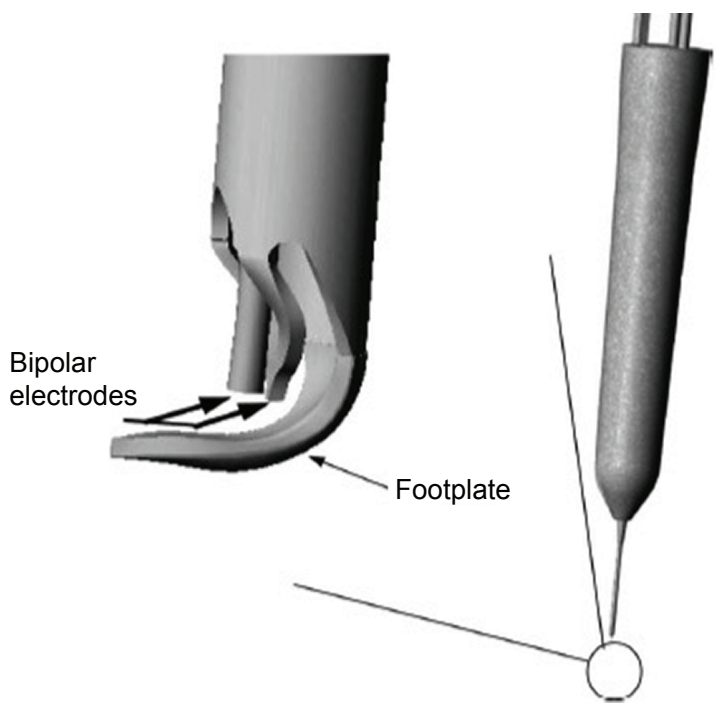

Figure I Trabectome handpiece showing the footplate which glides within Schlemm's canal and the bipolar electrodes which cauterize the inner wall of Schlemm's canal. Note: Reproduced from Francis BA, See RF, Rao NA, Minckler DS, Baerveldt G. Ab interno trabeculectomy: development of a novel device (Trabectome) and surgery for open-angle glaucoma. J Glaucoma. 2006;15:68-73." Copyright (C 2006. Promotional and commercial use of the material in print, digital or mobile device format is prohibited without the permission from the publisher Wolters Kluwer Health. Please contact healthpermissions@wolterskluwer.com for further information.

\section{Trabectome with CE/IOL}

In 2008, Francis et al described results of 304 patients undergoing Trabectome combined with cataract extraction/ intraocular lens implant (CE/IOL) who started with a mean IOP of $20.0 \pm 6.3 \mathrm{mmHg}$ and $2.7 \pm 1.1$ mean medications. At 1-year follow-up, the IOP was reduced to $15.5 \pm 2.9 \mathrm{mmHg}$ with $1.4 \pm 1.3$ medications at 12 months in the remaining cohort. The mean IOP reduction (calculated as mean of IOP reduction for each patient) was $15.6 \%$, but when calculated as the percent reduction of average final IOP from average baseline IOP (as most studies have done), the IOP reduction was $22.5 \%{ }^{14}$

\section{Trabectome with and without CE/IOL}

In 2008, Minckler et al also described the results available from post-marketing surveillance on the Trabectome. For Trabectome-only cases, IOP was reduced from $25.7 \pm 7.7 \mathrm{mmHg}$ to $16.6 \pm 4.0 \mathrm{mmHg}$ (40\% reduction) at 24 months, with number of medications reduced from 2.93 to 1.2. For Trabectome/CE/IOL cases, baseline IOP was reduced from $20.0 \pm 6.2 \mathrm{mmHg}$ to $15.9 \pm 3.3 \mathrm{mmHg}(18 \%$ reduction) at 12 months, with medications reduced from $2.63 \pm 1.12$ to $1.50 \pm 1.36 .{ }^{10}$ In addition to its retrospective nonrandomized nature, this study is limited by significant loss to follow-up at the follow-up periods.

In 2012, Ting et al compared the results of Trabectome in pseudoexfoliation glaucoma $(\mathrm{PXG})$ to those in primary $\mathrm{OAG}$ (POAG) in a prospective nonrandomized study. ${ }^{15}$ Among those that received Trabectome only, mean IOP was reduced from $29.0 \pm 7.5 \mathrm{mmHg}$ to $16.1 \pm 4.0 \mathrm{mmHg}(12.3 \pm 8.0 \mathrm{mmHg}$ reduction) in the PXG group at 1 year, and IOP was reduced from $25.5 \pm 7.9 \mathrm{mmHg}$ to $16.8 \pm 3.9 \mathrm{mmHg}(7.5 \pm 7.4 \mathrm{mmHg}$ reduction) in the POAG group at 1 year. Among those that received Trabectome/CE/IOL, mean IOP was reduced from $21.7 \pm 8.4 \mathrm{mmHg}$ to $14.2 \pm 3.2 \mathrm{mmHg}(7.2 \pm 7.7 \mathrm{mmHg}$ reduction) in the PXG group at 1 year and mean IOP was reduced from $19.9 \pm 5.4 \mathrm{mmHg}$ to $15.6 \pm 3.2 \mathrm{mmHg}(4.1 \pm 4.6$ reduction $)$ in the POAG group at 1 year. The authors hypothesized that PXG patients have more obstruction at the level of the TM, in part due to the mechanical blockage of exfoliative material, and that POAG patients may be more likely to have obstruction distal to the TM. ${ }^{15}$ In fact, there is evidence that simply the act of irrigating and aspirating the exfoliative material may contribute to IOP reduction in PXG patients. ${ }^{16}$

In 2013, Ahuja et al reported the single-surgeon, singlecenter results of OAG patients undergoing Trabectome or Trabectome/CE/IOL from the Mayo Clinic in Rochester, MN, USA. The authors reported that, at 24-month follow-up, Trabectome-only patients improved from $25.9 \pm 8.9 \mathrm{mmHg}$ to $16.8 \pm 2.8 \mathrm{mmHg}(35.1 \%$ reduction $)$ and Trabectome/CE/ IOL patients improved from $19.3 \pm 7.4 \mathrm{mmHg}$ to $14.9 \pm 4.9$ $\mathrm{mmHg}$ (22.8\% reduction). For both groups combined, $62 \%$ had achieved the success criteria of postoperative IOP $\leq 21$ $\mathrm{mmHg}$ or $\geq 20 \%$ reduction in IOP and not needing an increase in glaucoma medications or additional glaucoma surgery. ${ }^{17}$

Similarly, in 2013, Jordan et al described single-center, prospective observational results from Germany on POAG and PXG patients undergoing Trabectome or Trabectome/ $\mathrm{CE} / \mathrm{IOL}$. Results from the two types of procedures were not separated and were only reported combined. In the POAG group, IOP was improved from $24.5 \pm 5.5 \mathrm{mmHg}$ with $2.1 \pm 1.3$ medications to $18 \pm 6.1 \mathrm{mmHg}$ with $1.2 \pm 1.1$ medications $(26 \%$ IOP reduction with $43 \%$ medication reduction). In the PXG group, IOP was reduced from $25 \pm 5.9 \mathrm{mmHg}$ with $2.0 \pm 1.2$ medications to $18 \pm 8.2 \mathrm{mmHg}$ to $1.1 \pm 1.1$ medications $(28 \%$ IOP reduction with $45 \%$ medication reduction). The mean follow-up periods were $204 \pm 238$ days and $200 \pm 278$ days, respectively. ${ }^{18}$

\section{Adverse events and clinical considerations}

In the available case series, ${ }^{9-12,14,15,17,18}$ the most common complications after Trabectome were IOP spikes on postoperative day 1. While intraoperative blood reflux from Schlemm's canal is considered to be an expected part of surgery and generally resolves by week 1 , there have been reports of delayedonset hyphema (2-31 months following the procedure), and 
suggested triggers include Valsalva maneuver, use of aspirin and warfarin, and an IOP below episcleral venous pressure with physiologic blood reflux into the anterior chamber via an unroofed Schlemm's canal. ${ }^{19}$ Luebke et al showed, in a retrospective review of 137 patients at the University Eye Hospital in Freiburg, Germany, that visual outcomes were not statistically different in patients that had cataract surgery with and without Trabectome; however, the rate of cystoid macula edema was slightly higher $(2.2 \%$ in combined surgery vs $1.9 \%$ in cataract surgery alone). ${ }^{20}$ In the reported studies here, ${ }^{9-12,14,15,17,18}$ there have been no reports of infection, choroidal effusion, choroidal hemorrhage, or wound leaks.

Patients that respond best to Trabectome have normal episcleral venous pressure and IOP targets above episcleral venous pressure. In addition, the studies above generally had higher baseline IOPs in the Trabectome-only group compared to the Trabectome/CE/IOL group, and the difference in percent IOP reductions between these groups demonstrates that patients with higher baseline IOPs are likely to have a greater IOP reduction than those with lower baseline IOPs. While, as described above, Ting et al hypothesized that PXG patients may have a better IOP response to Trabectome than those with POAG, ${ }^{15}$ this differential effect was not seen by Jordan et al. ${ }^{18}$ Finally, Klamann et al explored in a retrospective comparative study whether prior SLT influenced Trabectome/CE/ IOL outcomes in OAG patients, and they reported that there was no adverse effect of prior SLT on Trabectome/CE/IOL outcomes, and, in fact, there was a slight though insignificant improvement in IOP reduction outcomes among the group that had previously received SLT. ${ }^{21}$

\section{iStent}

The iStent (Glaukos Corporation, Laguna Hills, CA, USA) is a first-generation trabecular micro-bypass product that directly connects the anterior chamber to Schlemm's canal and creates a permanent opening into Schlemm's canal (Figure 2A). It was FDA approved in 2012 when combined with cataract surgery in the US, and is CE marked both alone and in combination with cataract surgery in Europe.

The device is composed of a heparin-coated, nonferromagnetic, titanium stent, approximately $1 \times 0.3 \mathrm{~mm}$ in size, that connects at a right angle to the canal-implanted portion, which has a pointed end. It comes with an inserter, which is guided into a corneal wound, at least $1.7 \mathrm{~mm}$ in size, and into the anterior chamber under ophthalmic viscoelastic device (OVD). With the help of a surgical gonioscopy lens, it is implanted into Schlemm's canal with a sideways sliding technique that seats it there permanently.

There is also a second-generation model called the iStent inject (Glaukos Corporation), which has been CE marked for use in Europe (Figure 2B). ${ }^{22}$ It is a smaller titanium device, $360 \mu \mathrm{m}$ in length, and contains a head facing the anterior chamber that is $230 \mu \mathrm{m}$ in width with four inlets for the passage of aqueous into the device and out through Schlemm's canal. The inserter comes preloaded with two stents. It has the advantage of an easier surgical technique because sideways sliding of the stent is not required for positioning and because two devices can be injected at the same time without ever exiting the eye.

Like the Trabectome, the iStent reduces resistance in the juxtacanalicular TM. Hunter et al showed in anterior segment perfusion models with the iStent inject that outflow facility increases from $0.16 \pm 0.05 \mu \mathrm{L} / \mathrm{min} / \mathrm{mmHg}$ to $0.38 \pm 0.23$ $(P<0.03, \mathrm{n}=7)$, and then an additional iStent inject further increased outflow facility to $0.78 \pm 0.66(n=2) .{ }^{23}$ Similar results were reported from an ex vivo whole human eye model, with similar effects of a second iStent further lowering pressure from the initial iStent placed. ${ }^{24}$

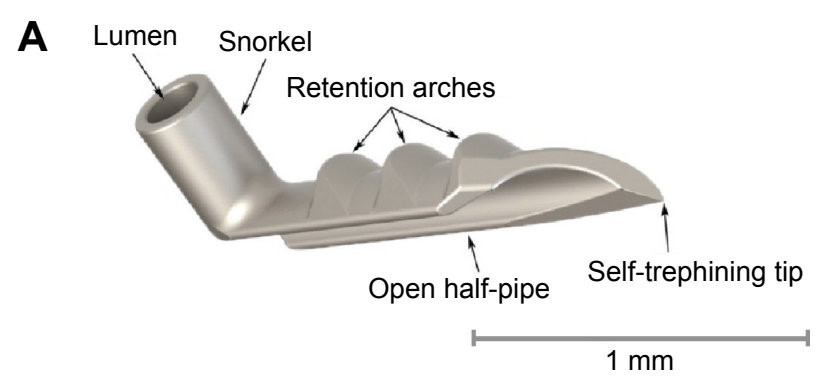

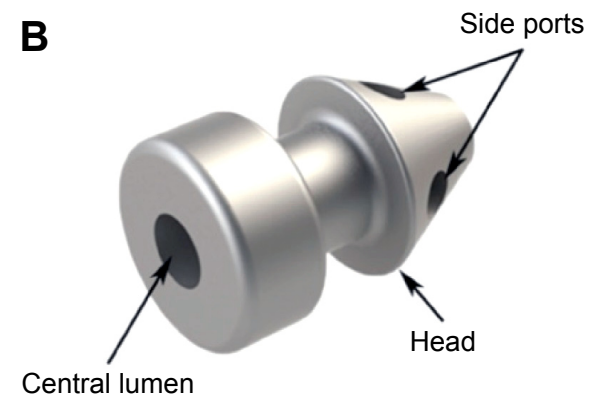

$1 \mathrm{~mm}$

Figure 2 The first-generation trabecular iStent (A) with the self-trephining tip that is inserted into Schlemm's canal via a sideways sliding technique, and then is maintained by the retention arches. The lumen is the portion facing the anterior chamber. The second-generation trabecular iStent inject (B), with four side ports within the Schlemm's canal. The central lumen faces the anterior chamber. This model eliminates the need for the sideways sliding movement in the surgery technique.

Note: Copyright (C) 2014. Dove Medical Press. Adapted from Hunter KS, Fjield T, Heitzmann H, Shandas R, Kahook MY. Characterization of micro-invasive trabecular bypass stents by ex vivo perfusion and computational flow modeling. Clin Ophthalmol. 2014;8:499-506. ${ }^{23}$ 


\section{Efficacy}

\section{iStent with $\mathrm{CE} / \mathrm{IOL}$}

In 2010, Fea performed a small prospective double-masked randomized clinical trial based at one center in Torino, Italy, comparing iStent plus cataract surgery to cataract surgery alone among patients with IOP $>18 \mathrm{mmHg}$ on at least one medication. Baseline IOP was $18.7 \pm 2.6 \mathrm{mmHg}$ in the stent/CE/IOL group and $17.3 \pm 3.0 \mathrm{mmHg}$ in the $\mathrm{CE} /$ IOL group. At 15 months, the stent/CE/IOL group had an IOP of $14.8 \pm 1.2 \mathrm{mmHg}$ compared to $15.7 \pm 1.1 \mathrm{mmHg}$ in the $\mathrm{CE} / \mathrm{IOL}$ group $(P=0.032)$. This resulted in a $17.3 \%$ reduction with $80 \%$ medication reduction in the stent/CE/IOL group at 15 months (compared to $9.2 \%$ IOP reduction and $31.6 \%$ medication reduction in the $\mathrm{CE} / \mathrm{IOL}$ group). After a 1 -month washout period, IOP was $16.6 \pm 3.1 \mathrm{mmHg}$ in the stent/CE/IOL group compared to $19.2 \pm 3.5 \mathrm{mmHg}$ in the $\mathrm{CE} /$ $\operatorname{IOL}$ group $(P=0.042){ }^{25}$

In 2011, Samuelson et al, for the US iStent Study Group, performed a prospective randomized controlled multicenter clinical trial, in which 240 eyes with mild-to-moderate glaucoma with IOP $\leq 24 \mathrm{mmHg}$ on one to three medications, with IOP between 22 and $36 \mathrm{mmHg}$ after washout of medications, were randomized to iStent combined with cataract surgery versus cataract surgery alone. Baseline IOP was $18.7 \pm 3.3 \mathrm{mmHg}$ on medications in the stent/CE/IOL group and $18.0 \pm 3.0 \mathrm{mmHg}$ in the $\mathrm{CE} / \mathrm{IOL}$ group $(25.2 \pm 3.5 \mathrm{mmHg}$ in stent/CE/IOL and $25.5 \pm 3.7$ in the $\mathrm{CE} / \mathrm{IOL}$ group after washout). Final IOP in each group at 12 months was not reported, but mean reduction (treated) from treated baseline was $1.5 \pm 3.0 \mathrm{mmHg}$ in the stent/CE/IOL group and $1.0 \pm 3.3$ $\mathrm{mmHg}$ in $\mathrm{CE} / \mathrm{IOL}$ ( $P$-value not reported). The percent IOP reduction was $8 \%$ with $87 \%$ medication reduction in the stent CE/IOL group at 12 months (compared to $5.5 \%$ IOP reduction and $73 \%$ medication reduction in the $\mathrm{CE} / \mathrm{IOL}$ group). At 1 year, $72 \%$ of the stent/CE/IOL group had unmedicated IOP $\leq 21 \mathrm{mmHg}$, compared to $50 \%$ of $\mathrm{CE} / \mathrm{IOL}$-group eyes $(P<0.001)$. Sixty-six percent of stent/CE/IOL-group eyes, compared to $48 \%$ of $\mathrm{CE} / \mathrm{IOL}$-group eyes, had $\geq 20 \%$ IOP reduction. ${ }^{26}$

In 2012, Craven et al, for the iStent Study Group, performed a prospective randomized controlled multicenter clinical trial with 2-year results that showed similar results. Eyes with mild-to-moderate glaucoma with IOP between 22 and $36 \mathrm{mmHg}$ were randomized to cataract surgery alone or in combination with the iStent. Baseline IOP on medications was $18.6 \pm 3.4 \mathrm{mmHg}$ in the stent/CE/IOL group and 17.9 \pm 3.0 $\mathrm{mmHg}$ in the $\mathrm{CE} / \mathrm{IOL}$ group $(25.4 \pm 3.5 \mathrm{mmHg}$ in stent/CE/ IOL and $25.2 \pm 3.6 \mathrm{mmHg}$ in $\mathrm{CE} / \mathrm{IOL}$ after washout). At 12 months, treated IOP was $17.0 \pm 2.8 \mathrm{mmHg}$ in stent/CE/IOL and $17.0 \pm 3.1 \mathrm{mmHg}$ in $\mathrm{CE} / \mathrm{IOL}$ ( $P$-value not reported). This resulted in an $8.6 \%$ IOP reduction with $88 \%$ medication reduction in the stent/CE/IOL group (compared to $5.0 \%$ IOP reduction and $73 \%$ medication reduction in the $\mathrm{CE} /$ IOL group). At 24 -month follow-up, $61 \%$ of the stent/CE/ IOL group maintained IOP $\leq 21 \mathrm{mmHg}$ without medication, compared to $50 \%$ of the $\mathrm{CE} / \mathrm{IOL}$ group $(P=0.036)$. Additionally, $53 \%$ of the stent/CE/IOL group had IOP reduction $\geq 20 \%$ without medication compared to $44 \%$ of the $\mathrm{CE} / \mathrm{IOL}$ group $(P=0.090)$. The mean IOP in the stent/CE/IOL group was $17.0 \mathrm{mmHg}$ after 1 year and $17.1 \mathrm{mmHg}$ after 2 years, compared to the $\mathrm{CE} / \mathrm{IOL}$ group, in which it was $17.0 \mathrm{mmHg}$ and $17.8 \mathrm{mmHg}$ during the same time period. There was no statistically significant difference in ocular hypotensive medication use between groups at 24 months. ${ }^{27}$

\section{Multiple iStents with CE/IOL}

Since small IOP effects have been demonstrated with the use of one iStent, attention has turned towards using multiple iStents, and this is, in fact, the approach of the secondgeneration iStent inject, which comes in a package of two iStents.

In 2010, Fernández-Barrientos evaluated changes in aqueous humor dynamics as well as IOP outcomes of patients undergoing two iStents with concurrent cataract surgery compared to cataract surgery alone in 33 eyes. Aqueous outflow facility increased by $275 \%$ in the treatment group compared to $46 \%$ in the control group after 12 months $(P<0.02)$, and the mean IOP reduction was $6.6 \mathrm{mmHg}$ compared to $3.9 \mathrm{mmHg}$ in the control group $(P<0.002)$, with medication use of 0.0 versus $0.7(P<0.007)$ in the treatment versus control group, respectively. This resulted in a $27 \%$ IOP reduction with $91 \%$ medication reduction in the two-stent/CE/IOL group (compared to 16\% IOP reduction with $42 \%$ medication reduction in the $\mathrm{CE} / \mathrm{IOL}$ group). This small study suggests a larger treatment effect in those receiving two iStents, as compared to one. ${ }^{28}$

In 2012, Belovay et al reported on a comparative case series also using multiple iStents from a single surgeon in Ontario, Canada, in which 53 eyes underwent implantation of two or three iStents with concurrent cataract surgery. Mean IOP at baseline was $18.0 \pm 4 \mathrm{mmHg}$, and patients were on $2.7 \pm 1.0$ medications at baseline. At 1 year postoperatively, mean IOP had decreased to $14.3 \pm 2.9 \mathrm{mmHg}$, and they were on $0.7 \pm 1.1$ medications. At 1 year, there was an $83 \%$ reduction in the number of topical ocular hypotensive patients compared to preoperatively. The three-stent group was, on average, on 0.4 medications compared to one medication in the two-stent group. Overall, there was a $20 \%$ IOP reduction 
with $64 \%$ medication reduction in the two-stent/CE/IOL group, compared to a $20 \%$ IOP reduction with $85 \%$ medication reduction with three stents/CE/IOL. ${ }^{29}$

\section{Multiple iStents alone}

In 2014, Voskanyan et al reported results of the Synergy trial, using the second-generation iStent inject among 99 phakic and pseudophakic subjects. Their results showed a 28.9\% IOP reduction from medicated baseline, but the final mean medication usage was not reported. At 1 year after implantation of two stents, $66 \%$ had IOP $\leq 18 \mathrm{mmHg}$ off of medication, and the mean IOP reduction among all was $40 \%{ }^{22}$

Similarly, in the same year, Fea et al compared the iStent inject, which consists of two stents per eye, to two ocular hypotensive agents in a mostly phakic group of patients with POAG in a multicenter European trial. Their results demonstrated a 48\% IOP reduction in the iStent inject (two stents) group, as compared to a $46.7 \%$ IOP reduction in the two-medications (fixed combination medication of latanoprost and timolol) group. Both groups had significant IOP reduction, with $94.7 \%$ of the stent group and $91.8 \%$ of the medication group achieving an IOP reduction of $\geq 20 \%$. The mean IOP reduction was $8.1 \pm 2.6 \mathrm{mmHg}$ in the stent group compared to $7.3 \pm 2.2 \mathrm{mmHg}$ in the medication group, suggesting that the iStent inject is at least as effective as two medications at 1 -year follow-up. ${ }^{30}$

In 2015, Klamann et al reported the 6-month iStent inject outcomes of a retrospective group of 35 consecutive phakic eyes with moderate POAG, PXG, or pigmentary glaucoma not well controlled with medications. Among patients with POAG and PXG, the IOP was decreased 33\% and $35 \%$, respectively, at 6 -month follow-up. Additionally, the medications were reduced from $2.19 \pm 0.91$ to $0.88 \pm 0.62$ (60\% medication reduction) in the POAG group and from $2.33 \pm 1.23$ to $1.04 \pm 0.30$ ( $55 \%$ medication reduction) in the PXG group. However, in the pigmentary glaucoma group $(n=3)$, within 4 weeks after surgery and 7 days after cessation of steroids, IOP was $>30 \mathrm{mmHg}$ despite an increase in ocular hypotensive medications. These patients subsequently underwent trabeculectomy. This small study showed a poor IOP outcome in phakic pigmentary glaucoma patients, but larger studies are needed to confirm this observation. ${ }^{31}$

\section{Adverse events and clinical considerations}

As with other trabecular procedures, intraoperative blood reflux from Schlemm's canal is a normal part of the surgery. The most common complications were minor and were related to stent malposition or temporary obstruction, which were alleviated with secondary procedures such as YAG synechiolysis or secondary stent implantation. ${ }^{22-31}$ As described above, three of three phakic pigmentary glaucoma patients in a small report were shown to have worsening of IOP to $>30 \mathrm{mmHg}$ despite an increase in ocular hypotensive medications, ${ }^{31}$ but larger studies are needed to confirm pigmentary glaucoma as a relative contraindication to iStent surgery. As with all trabecular procedures, elevated episcleral venous pressure is a relative contraindication, along with lower starting IOPs. Finally, the current studies suggest that concurrent implantation of two stents will provide a significantly greater IOP reduction, more comparable to the Trabectome, than implantation of just one stent.

\section{Hydrus}

The Hydrus Microstent (Ivantis, Inc, Irvine, CA, USA) is an $8 \mathrm{~mm}$-long, crescent-shaped open structure, curved to match the shape of Schlemm's canal (Figure 3). It is made of nitinol (nickel-titanium alloy), which is a shape memory alloy. This means that when deformed, it returns to its original shape after being heated. It uses a preloaded injector via a clear corneal incision and is inserted into and sits within Schlemm's canal, in which it extends 3 clock hours. It does not block the collector channel ostia in the posterior portion

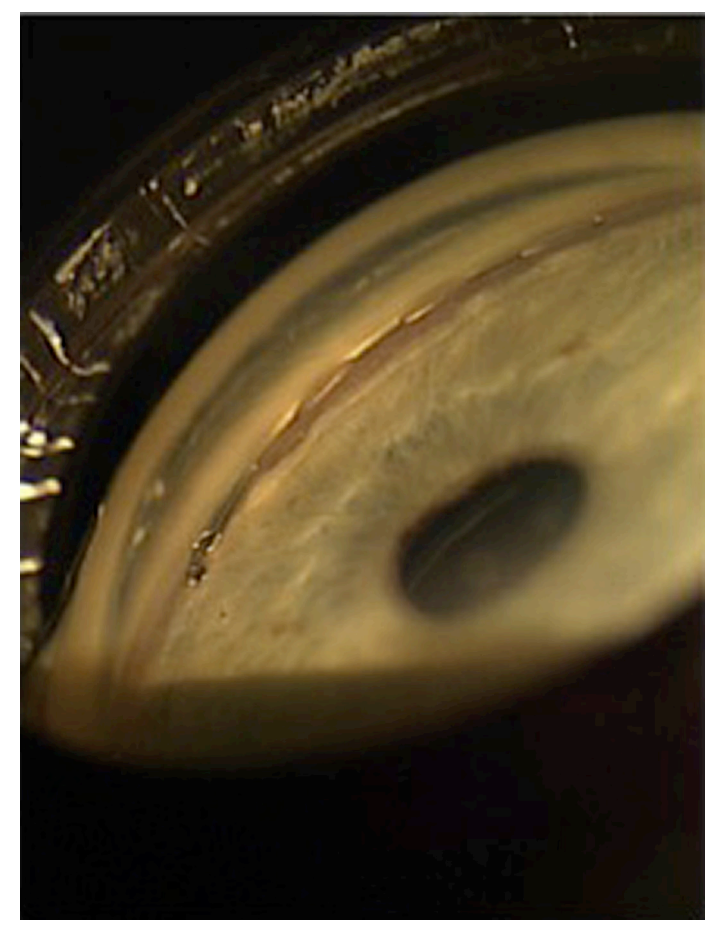

Figure 3 The Hydrus trabecular stent sitting within a dilated Schlemm's canal. Note: Reprinted from Grover DS, Godfrey DG, Smith O, Feuer WJ, Montes de Oca I, Fellman RL. Gonioscopy-assisted transluminal trabeculotomy, ab interno trabeculotomy: technique report and preliminary results. Ophthalmology. 2014; |21:855-861. ${ }^{34}$ Copyright (C) 2014, with permission from Elsevier. 
of Schlemm's canal because it has a scaffold design, and it has a $1 \mathrm{~mm}$ inlet portion, which resides within the anterior chamber. When not inserted into a standard cataract corneal wound, it can also be inserted through a $1-1.5 \mathrm{~mm}$ corneal incision. Once inserted into Schlemm's canal, it can dilate it by four to five times the natural width of the canal. The rationale behind dilating Schlemm's canal lies in the previous findings that elevated IOP actually causes the canal to collapse, leading to lasting changes in the TM and adjacent Schlemm's canal. ${ }^{32}$ This is also the rationale behind canaloplasty, a procedure that also aims to dilate the Schlemm's canal; canaloplasty is not conjunctival-sparing and thus is not discussed in this review.

\section{Efficacy}

Hydrus with $\mathrm{CE} / \mathrm{IOL}$

The Hydrus has received the CE mark in Europe and is under clinical investigation in the United States. Pfeiffer et al recently reported the initial 2-year randomized controlled single-masked clinical trial results of the Hydrus Microstent with concurrent cataract surgery compared to cataract surgery alone. This study involved a washout of glaucoma medications and outcomes were reported without the influence of concomitant glaucoma medications. The washed-out baseline IOP was $26.3 \pm 4.4 \mathrm{mmHg}$ in the Hydrus/CE/IOL group and $26.6 \pm 4.2 \mathrm{mmHg}$ in the $\mathrm{CE} / \mathrm{IOL}$ group. At 24-month follow-up, the IOP was $16.9 \pm 3.3 \mathrm{mmHg}$ in the Hydrus/CE/ IOL group and 19.2 \pm 4.7 in the $\mathrm{CE} / \mathrm{IOL}$ group. At 2 years, the authors reported that $80 \%$ of Hydrus patients had a $20 \%$ reduction in washed-out IOP compared to $46 \%$ of patients undergoing cataract surgery alone $(P=0.0008)$. The IOP in each group was $16.9 \pm 3.3 \mathrm{mmHg}$ in the Hydrus group as compared to $19.2 \pm 4.7 \mathrm{mmHg}$ in the controls $(P=0.0093) .{ }^{33}$

\section{Adverse events and clinical considerations}

The safety in the Hydrus group was equivalent to the control. Six of 50 (12\%) Hydrus patients did have focal peripheral anterior synechiae, but this had no correlation with device efficacy. ${ }^{33}$ On a theoretical basis, the Hydrus stent will have the same indications and relative contraindications as the iStent, but more data are needed to support this idea.

\section{GATT}

GATT is another form of ab interno trabeculotomy recently described in 2014 (Figure 4). This technique was developed by Grover et al at Glaucoma Associates of Texas and is also sutureless and conjunctival-sparing. ${ }^{34}$ Two paracentesis wounds are created - one temporally and the other at the
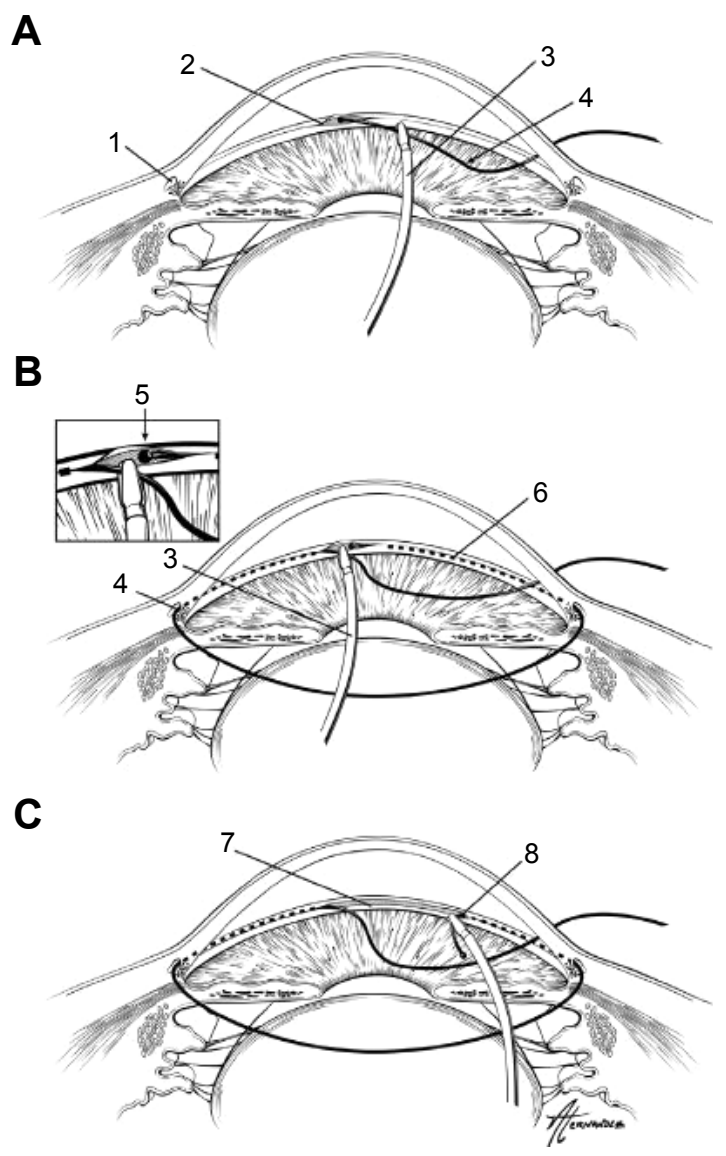

Figure 4 Demonstration of gonioscopy-assisted transluminal trabeculotomy. Notes: An illuminated microcatheter is inserted through a nasal paracentesis wound (A). After a small goniotomy wound is created, the microcatheter is inserted into Schlemm's canal using microsurgical forceps. After being guided around $360^{\circ}$ (B), each end of the microcatheter is externalized from the temporal wound, creating a $360^{\circ}$ trabeculotomy $(\mathbf{C})$. I=Schlemm's canal; $2=$ =initial goniotomy site; $3=$ =microsurgical forceps; $4=$ microcatheter; $5=$ distal end of microcatheter after being passed $360^{\circ}$ around Schlemm's canal; $6=$ path of microcatheter within Schlemm's canal; $7=$ trabecular shelf created after this procedure; $8=$ trabeculotomy created when the distal end of catheter is externalized. Reprinted from Pfeiffer N, Garcia-Feijoo J, Martinez-de-la-Casa JM, et al. A randomized trial of a Schlemm's canal microstent with phacoemulsification for reducing intraocular pressure in open-angle glaucoma. Ophthalmology. 2015;122:1283-1293. ${ }^{33}$ Copyright (C) 2015, with permission from Elsevier.

superonasal or inferonasal quadrant - and a OVD is injected to maintain anterior chamber stability. An illuminated microcatheter is inserted through the nasal paracentesis wound. Under the guidance of a gonioscopy lens, a 1-2 mm goniotomy is created in the nasal TM angle using a microsurgical blade. Microsurgical forceps are then inserted into the temporal wound and used to insert the microcatheter (iTrack; Ellex, Adelaide, Australia) into the goniotomy incision. The forceps are used to advance the catheter circumferentially $360^{\circ}$ and the catheter's progress can be followed by the illuminated tip. Once the distal tip of the catheter makes it all the way back to the initial goniotomy incision, it can then be externalized through the temporal paracentesis. This creates the first $180^{\circ}$ of the trabeculotomy. Then the proximal tip of 
the catheter is brought to the temporal paracentesis, completing the $360^{\circ}$ trabeculotomy. Irrigation and aspiration are used to remove OVD and blood from the anterior chamber, and a $25 \%$ OVD fill is left at the end of the procedure. When combined with CE/IOL, GATT is performed first. ${ }^{34}$

This procedure offers an ab interno modification of $360^{\circ}$ suture trabeculotomy, which is typically performed $a b$ externo with a conjunctival peritomy and a scleral flap to access Schlemm's canal. It was first described for treatment of primary congenital glaucoma, but has more recently been reported for adult $\mathrm{OAG}$. $^{35,36}$

\section{Efficacy}

\section{GATT with and without CE/IOL}

In 2014, Grover et al reported the initial retrospective case series of 85 eyes with POAG and other secondary glaucomas undergoing GATT both with and without CE/IOL with 6 months of follow-up. Among the 55 POAG eyes, IOP decreased by $7.7 \pm 6.2 \mathrm{mmHg}$ with a $30 \% \pm 20 \%$ reduction in IOP, and an average decrease in glaucoma medications of $0.9 \pm 1.3$ at 12 months. In the remaining secondary glaucoma eyes, IOP was reduced by $17.2 \pm 10.8 \mathrm{mmHg}$, with a $56.8 \% \pm 17.4 \%$ reduction, and a decrease in glaucoma medications of $1.9 \pm 2.1$ at 12 months. ${ }^{34}$

More recently, Grover et al reported preliminary results of GATT for treating primary congenital glaucoma and juvenile OAG. In 14 eyes with at least 12 months of follow-up, IOP was reduced from a mean of 27.3 to $14.8 \mathrm{mmHg}$, and medications were reduced from 2.6 to $0.86 .{ }^{37}$

The theoretical advantage of the GATT procedures is that $360^{\circ}$ of the angle are treated, as opposed to a smaller portion with Trabectome, iStent, or even conventional goniotomy or ab externo trabeculotomy. In comparison to the Trabectome, which ablates the inner wall of Schlemm's canal using electrocautery, GATT has the end result of a conventional trabeculotomy with a "trabecular shelf" of tissue remaining. ${ }^{34}$ Long-term outcomes of late failure are not yet known, and it remains to be seen if scarred closure of this shelf will be more likely in GATT as compared to the electrocauterized shelf of post-Trabectome eyes.

\section{Adverse events and clinical considerations}

As with Trabectome, the most common postoperative event was a hyphema, which was still present in $30 \%$ of patients at the 1 -week postoperative visit, but all resolved at 1 month. ${ }^{34}$ There was one case of anterior chamber shallowing and one case of transient choroidal folds, both of which resolved by the month 1 visit. According to the initial experience of Grover et al, ${ }^{34}$ absolute contraindications include an inability to stop anticoagulation medication, a bleeding diathesis, an unstable IOP, a closed angle, and inability to identify TM. Theoretically, the indications of GATT should be similar to those for the Trabectome, but future studies should verify this idea.

\section{ELT}

ELT is another form of ab interno trabeculotomy that is sutureless and spares the conjunctiva. ELT, as performed with an $\mathrm{XeCl} 308 \mathrm{~nm}$ laser, was first described in 1987 by Berlin et al in the laboratory. ${ }^{38}$ It utilizes a photoablative approach that vaporizes TM, resulting in a cooling effect on the tissue, thus limiting thermal damage. The first device uses a laser probe (AIDA; Glautec AG, Nürnberg, Germany) with the assistance of a gonioscopy lens to visualize the procedure, and the second has an endoscopic laser probe (AIDA; TUILaser, Munich, Germany). The first device uses a $1.2 \mathrm{~mm}$ incision that is made either temporally or nasally, and OVD is injected into the anterior chamber. The laser probe is inserted into the eye and held tip-up $2 \mathrm{~mm}$ from the TM as a gonioscopy lens is applied to the cornea and used for visualization. Then the laser tip is brought directly into contact with the TM, and eight to ten laser spots, equally distributed approximately $500 \mu \mathrm{m}$ from one another, are used to puncture and create small holes in the anterior trabecular meshwork. With the second device, gonioscopy is not necessary, and visualization of the anterior trabecular meshwork for treatment is possible via the endoscopic laser probe. Perforations with microbleeding can be seen after successful treatment with the excimer laser. The laser has a wavelength of 308 $\mathrm{nm}$, spot size of $200 \mu \mathrm{m}$, and pulse energy of $1.2 \mathrm{~mJ}$ with a duration of $80 \mathrm{~ns}$.

\section{Efficacy}

\section{ELT alone}

Babighian et al conducted a prospective randomized controlled 2-year study comparing $180^{\circ}$ of treatment by ELT (with gonioscopy lens) versus SLT. Baseline IOP was $25.0 \pm 1.9 \mathrm{mmHg}$ in the ELT group and $23.9 \pm 0.9 \mathrm{mmHg}$ in the SLT group. Final IOP at 24 months was $17.6 \pm 2.2 \mathrm{mmHg}$ $(29.6 \% ; P<0.0001)$ in the ELT group and $19.1 \pm 1.8 \mathrm{mmHg}$ $(21 \% ; P<0.0001)$ in the SLT group. Mean IOP was reduced $29.6 \%$ in the ELT group versus $21 \%$ in the SLT group. Glaucoma medications were reduced from $2.27 \pm 0.7$ to $0.87 \pm 0.8$ in the ELT group compared to a reduction from $2.20 \pm 0.7$ to $0.87 \pm 0.8$ in the SLT group. Success rates, defined by $\geq 20 \%$ IOP reduction without additional glaucoma intervention, were $53.3 \%$ for the ELT group compared to $40 \%$ for the SLT group $(P=0.35) .{ }^{39}$ 


\section{ELT with CE/IOL}

Töteberg-Harms et al described results of a series of 28 patients who underwent endoscopic ELT combined with $\mathrm{CE} / \mathrm{IOL}$. The authors divided the group into those with baseline IOP above $21 \mathrm{mmHg}$ or $21 \mathrm{mmHg}$ and below (25.8 $\pm 2.9 \mathrm{mmHg}$ versus $16.5 \pm 2.9 \mathrm{mmHg}$ at baseline). At 12 months, IOP was reduced by $9.5 \pm 5.4 \mathrm{mmHg}$ in the higher baseline IOP group compared to $1.1 \pm 1.4 \mathrm{mmHg}$ in the lower baseline IOP group. This resulted in a $36.6 \%$ IOP reduction with $30 \%$ medication reduction in the higher baseline IOP group, compared to an $11.5 \%$ IOP reduction with $43 \%$ medication reduction in the lower baseline IOP group. Overall, in both groups, at 12 months, IOP was reduced by $4.5 \pm 5.9 \mathrm{mmHg}$, which was a $23 \%$ reduction in IOP, and glaucoma medications were reduced by $0.9 \pm 1.5 .^{40}$

\section{Adverse events and clinical considerations}

Microbleeding can occur intraoperatively after the laser is applied. In the available studies, no serious adverse events were reported. ${ }^{38-40}$ Additionally, IOP spikes were not mentioned. It remains to be seen whether studies with longer follow-up will demonstrate whether these small laser punctures in the anterior trabeculum are at greater risk of scarring down compared to the trabecular procedures with a stent or creation of a longer opening. Töteberg-Harms et al demonstrated that patients with higher baseline IOPs have a substantially greater IOP reduction response with ELT/CE/ IOL than those with lower baseline IOPs. ${ }^{40}$ Like the other trabecular procedures, this procedure is best for those with normal episcleral venous pressure (EVP) and baseline IOP higher than EVP.

\section{Increasing uveoscleral outflow: suprachoroidal shunts}

Uveoscleral outflow is thought to account for up to $50 \%$ of aqueous drainage in normal human eyes, ${ }^{41}$ and one study suggests that there is a negative pressure gradient of 3-4 $\mathrm{mmHg}$ that provides a potential driving force for aqueous outflow into the suprachoroidal space. ${ }^{42}$ The hypotensive effect of opening the uveoscleral pathway is exemplified by the use of prostaglandin analogs or a cyclodialysis cleft, which causes great IOP reduction. Suprachoroidal shunts direct aqueous outflow in a controlled fashion to the suprachoroidal space, essentially increasing the physiologic uveoscleral pathway. Disuse trabecular atrophy is a theoretical risk in this surgery type.

The Cypass (Transcend Medical, Menlo Park, CA, USA) is a suprachoroidal shunt, reviewed below. The iStent supra is another one under investigation, but there are no peer-reviewed studies to date. Finally, the SOLX Gold microshunt (SOLX, Waltham, MA, USA) is a suprachoroidal shunt implanted via ab externo conjunctival and scleral dissection and thus will not be discussed here. It is worth noting that while there are no published clinical trials on the SOLX Gold, it may be an alternative for a patient with corneal opacities precluding gonioscopy.

\section{Cypass}

The Cypass supraciliary micro-stent targets the suprachoroidal space and was granted the CE mark in 2008 (Figure 5). It is still under investigation in the United States.

The Cypass is a polyamide implant, $6.35 \mathrm{~mm}$ in length and $510 \mu \mathrm{m}$ in external diameter, that creates a permanent conduit between the anterior chamber and the supraciliary space. Along the length of the stent are microholes that allow for circumferential egress of aqueous into the suprachoroidal space, and the distal end of the stent allows longitudinal egress of fluid. Proximally, the collar of the device rests in the anterior chamber angle. Acetylcholine is injected to achieve miosis, and OVD is used to maintain chamber stability. The procedure is visualized using a gonioscopy lens. The Cypass is threaded onto a retractable guidewire of the applier. Through a $1.5 \mathrm{~mm}$ wound, the non-incisional tip of the guidewire is inserted and performs blunt dissection to create a plane between the ciliary body and the sclera. The micro-stent is then inserted into the dissected area until it reaches the supraciliary space, and then retention features are engaged. OVD is removed via standard irrigation and aspiration. Implant positioning could be confirmed by postoperative gonioscopy and/or anterior segment OCT. ${ }^{43}$

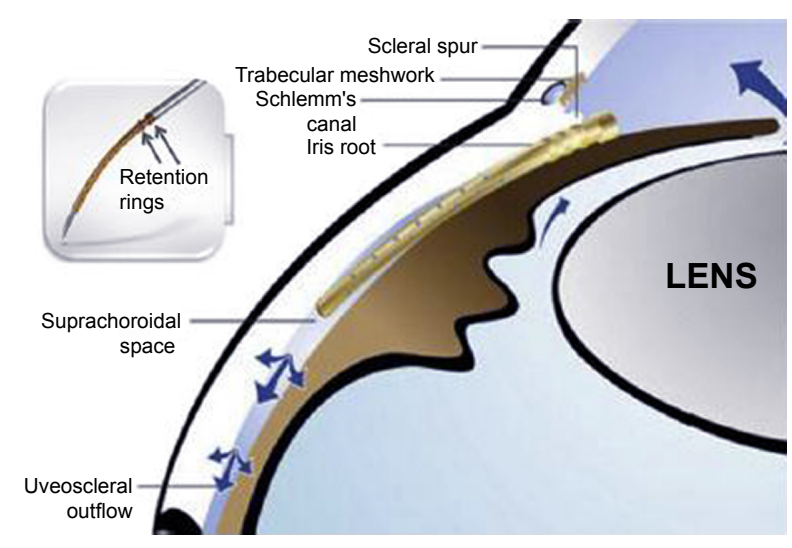

Figure 5 The Cypass suprachoroidal shunt in position in the suprachoroidal space with retention rings near the ciliary body face.

Notes: The blue arrows demonstrate the directional flow of aqueous. Reprinted from Hoeh H, Ahmed IK, Grisanti S, et al. Early postoperative safety and surgical outcomes after implantation of a suprachoroidal micro-stent for the treatment of open-angle glaucoma concomitant with cataract surgery. J Cataract Refract Surg. 2013;39:431-437. ${ }^{44}$ Copyright (C) 2013, with permission from Elsevier. 


\section{Efficacy}

\section{Cypass with $\mathrm{CE} / \mathrm{IOL}$}

In 2013, Hoeh et al reported the results of a multicenter prospective series of 57 uncontrolled $(\geq 21 \mathrm{mmHg}$ ) POAG patients and 41 IOP-controlled $(<21 \mathrm{mmHg})$ POAG patients undergoing Cypass implantation combined with cataract surgery. They demonstrated a favorable safety profile. The mean medicated IOP in both groups combined was 21.1 $\pm 5.91 \mathrm{mmHg}$ (baseline IOP for each group was not stated). The resulting IOP at 6 months was $15.6 \pm 0.53 \mathrm{mmHg}$ on $0.9 \pm 0.15$ medications in the uncontrolled group; this was a $37 \%$ decrease in IOP $(P<0.001)$ and a $50 \%$ reduction in glaucoma medications $(P<0.001)$. The resulting IOP in IOP-controlled patients was $15.6 \pm 0.68 \mathrm{mmHg}$ on $0.6 \pm 0.07$ medications; this was a $71.4 \%$ reduction in glaucoma medications $(P<0.001){ }^{44}$

\section{Cypass alone}

In a recent multicenter, single-arm interventional study, García-Feijoo et al reported the 1-year results of 65 eyes with OAG and IOP uncontrolled at $>21 \mathrm{mmHg}$ on topical therapy. Baseline IOP was reduced from $24.5 \pm 2.8 \mathrm{mmHg}$ with $2.2 \pm 1.1$ medications to $16.4 \pm 5.5 \mathrm{mmHg}$ with $1.4 \pm 1.3$ medications at 12 months. This was a $34.7 \%$ reduction in IOP. Eighty-three percent of eyes avoided conventional incisional glaucoma surgery. ${ }^{45}$

\section{Adverse events and clinical considerations}

There were no cases of serious postoperative events such as hypotony maculopathy, choroidal detachment, retinal detachment, or endophthalmitis in either of the two studies. ${ }^{44,45}$ In the study by Hoeh et al, early hypotony (13.8\%) and transient IOP increase (10.5\%) were the main complications; additionally, $4.4 \%$ had postoperative inflammation that persisted longer than 1 month. Peripheral anterior synechiae or partial obstruction of the stent were seen in two cases when stent implant alone was performed. ${ }^{45}$ García-Feijoo et al also reported that IOP spikes to $>30 \mathrm{mmHg}$ that lasted beyond 1 month occurred in $11 \%$ of cases; $12.2 \%$ were noted to have cataract progression at 12 months; and there were four eyes that had hyphema that resolved by month $1 .{ }^{45}$ Additional data will be needed to determine which patients respond best to a suprachoroidal shunt implantation.

\section{Reducing aqueous production: endocyclophotocoagulation}

Cyclophotocoagulation, initially via the transscleral approach, has long been used for refractory glaucoma. Recent clinical reports ${ }^{46,47}$ demonstrate the safety and efficacy of endocyclophotocoagulation (ECP; also called endoscopic photocoagulation) in the treatment of mild-to-moderate glaucoma. Like other minimally invasive glaucoma techniques, it is conjunctival-sparing, blebless, and can be combined with cataract surgery.

The curved laser endoscope probe (Endo Optiks, Little Silver, NJ, USA) can be inserted through a temporal $2.4 \mathrm{~mm}$ clear corneal wound into the OVD-filled anterior chamber and sulcus space (Figure 6). With direct endoscopic visualization of the anterior ciliary processes, the visible portion of the ciliary process epithelium is treated with the connected $810 \mathrm{~nm}$ diode laser (Iridex Oculight, Mountain View, CA, USA) at 200-400 $\mathrm{mW}$ continuous duration. Usually, approximately $270^{\circ}-360^{\circ}$ of anterior ciliary processes are treated to the point of blanching and shrinking of the tissue. Overtreatment is defined by a popping sound, which signifies tissue explosion.

\section{Efficacy ECP with CE/IOL}

In 2014, Francis et al reported the 3-year outcomes of a prospective nonrandomized matched-control study comparing ECP with $\mathrm{CE} / \mathrm{IOL}$ versus $\mathrm{CE} / \mathrm{IOL}$ alone in medically controlled OAG. The authors showed that the combined group $(n=80)$ had a baseline IOP of $18.1 \pm 3.0$ that was reduced to $16.0 \pm 3.3 \mathrm{mmHg}$ at 2 years with medications reduced from $1.5 \pm 0.8$ to $0.4 \pm 0.7$ (10.1\% IOP reduction with $73 \%$ medication reduction). The control group had a baseline IOP of $18.01 \pm 3.0$ that was reduced to $17.3 \pm 3.2 \mathrm{mmHg}$ at 2 years, and the control group had $2.4 \pm 1.0$ medications at baseline and $2.0 \pm 1.0$ medications at 2 years $(0.8 \%$ IOP reduction and $17 \%$ medication reduction). There was no difference in complications between the two groups. ${ }^{46}$

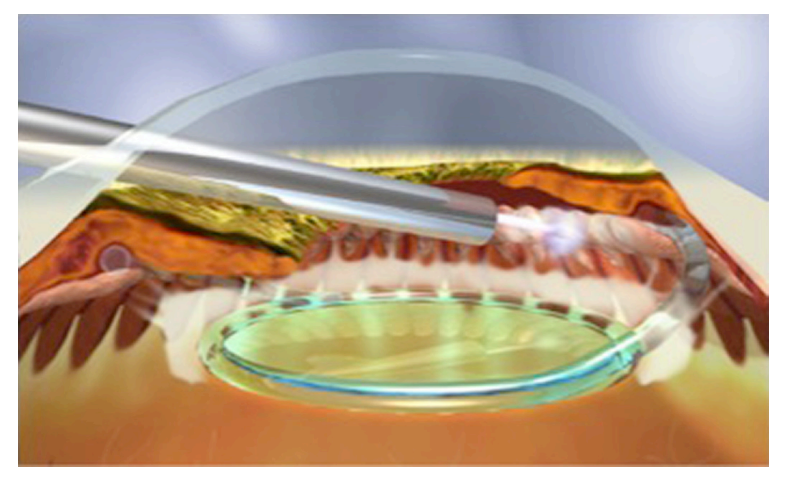

Figure 6 Endocyclophotocoagulation being performed on a pseudophakic patient. Note: Copyright (c) 2015. John Wiley and Sons. Reproduced from Siegel MJ, Boling WS, Faridi OS, et al. Combined endoscopic cyclophotocoagulation and phacoemulsification versus phacoemulsification alone in the treatment of mild to moderate glaucoma. Clin Experiment Ophthalmol. 2015;43:531-539.47 
In 2015, Siegel et al reported their retrospective results of $\mathrm{ECP}$ and $\mathrm{CE} / \mathrm{IOL}$ versus CE/IOL alone in mild-to-moderate glaucoma patients. They typically performed $270^{\circ}-300^{\circ}$ of treatment. Their ECP/CE/IOL group had a baseline IOP of $17.2 \pm 4.8$ on $1.3 \pm 0.6$ medications, which was reduced to $14.7 \pm 3.1 \mathrm{mmHg}$ on $0.2 \pm 0.6$ medications at 36 months $(14.5 \%$ IOP reduction with $85 \%$ medication reduction). Their $\mathrm{CE} /$ IOL group had a baseline IOP of $17.7 \pm 4.4$ on $1.5 \pm 0.7$ medications, which was reduced to $15.5 \pm 3.6 \mathrm{mmHg}$ on $1.3 \pm 0.6$ medications at 36 months. While there was no significant difference in the IOP reduction between groups $(P=0.34)$, there was a significant difference in medication reduction $(P<0.001)$. The authors reported, at 36 months, that $61.4 \%$ of the combined group versus $23.3 \%$ of the CE/IOL group achieved an outcome of $20 \%$ IOP reduction with a decrease of at least one ocular hypotensive medication. ${ }^{47}$

\section{Adverse events and clinical considerations}

Francis et al reported two patients developed an anterior chamber hemorrhage, but no cases of cystoid macular edema (CME) or any serious adverse events in the treatment group. ${ }^{46}$

Siegel et al reported four cases of CME (compared to one in the control group), two retinal detachments, and one case requiring penetrating keratoplasty in the treatment group. ${ }^{47}$

Interestingly, Tan et al recently reported on the application of ECP to perform endoscopic cycloplasty with $\mathrm{CE} /$ IOL in patients with severe plateau iris syndrome. In the 12 reported eyes, the anterior chamber angle was significantly opened, and thus they were effectively able to reverse the anatomic cause of angle closure, which $\mathrm{CE} / \mathrm{IOL}$ alone usually cannot do for these patients. ${ }^{48}$ Finally, it is worth noting that, in more refractory cases, ECP has also recently been described by Francis et al to work with increased IOPlowering when also performed through the pars plana (called "ECP-Plus" by Francis et al) to treat the posterior portion of the ciliary processes. This achieves a $61 \%$ IOP reduction but is currently reserved for refractory glaucoma due to increased risk for inflammation. ${ }^{49}$ More clinical data are needed, but it is likely that patients with other risk factors for CME, such as diabetes or uveitis, are not ideal candidates for ECP.

\section{Subconjunctival filtration: XEN gel stent}

Subconjunctival filtration creates a nonphysiologic route for aqueous outflow and is the basis of the traditional trabeculectomy and aqueous shunt glaucoma surgeries. The XEN gel

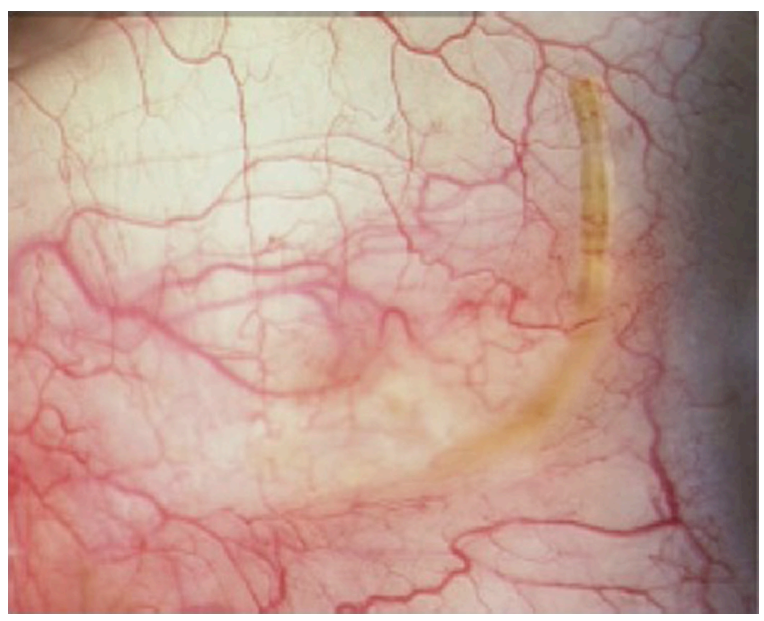

Figure 7 External view of the XEN subconjunctival gel stent in place.

Note: Reprinted from Lewis RA. Ab interno approach to the subconjunctival space using a collagen glaucoma stent. J Cataract Refract Surg. 2014;40:1301-1306. ${ }^{50}$ Copyright (c) 2014 with permission from Elsevier.

stent (Aquesys, Inc., Aliso Viejo, CA, USA) is an ab interno gelatin stent under investigation that would be implanted via a clear corneal incision without conjunctival dissection (Figure 7). The stent is $6 \mathrm{~mm}$ in length and composed of porcine gelatin crosslinked with glutaraldehyde. There are three models being evaluated that have inner diameters of $45 \mu \mathrm{m}, 63 \mu \mathrm{m}$, and $140 \mu \mathrm{m} .{ }^{50}$ The stent follows Poiseuille's law of laminar flow where the length of the tube and inner diameter of the tube manage the rate of flow. Hypotony is avoided by the flow resistance determined by the length and inner diameter of the tube. This procedure will rely on an eye that does not have prior conjunctival scarring, and it remains to be determined whether disuse trabecular atrophy could limit IOP outcomes in this type of surgery. While the $\mathrm{XEN}$ gel stent has a CE mark, there are not yet peer-reviewed published human clinical trials to date on its efficacy. ${ }^{50}$

\section{Discussion}

MIGS technology has the potential to solve a variety of problems in current glaucoma management. These include minimizing patient adherence problems, increasing quality of life for patients with ocular toxicity, and potentially reducing lifetime costs of expensive glaucoma medications, all while preserving the conjunctiva if additional, more invasive glaucoma surgeries are necessary in the future. Nonadherence rates in glaucoma have been reported to vary from $24 \%$ to $59 \%,{ }^{51}$ and patient reasons for nonadherence include forgetfulness, side effects, lack of affordability, difficulty administering drops, complicated medication schedules, poor understanding of the disease, and poor patient-doctor communication. ${ }^{52-54}$ Moderate-to-severe 
ocular surface disease is present in $71 \%$ of patients receiving triple drop therapy, ${ }^{53-56}$ and in these patients, implementing preservative-free alternatives may help but present additional cost and/or logistical insurance coverage barriers. Stein et al recently reported that laser trabeculoplasty is more costeffective than a prostaglandin analog for newly diagnosed POAG when taking into account realistic patient adherence rates. ${ }^{57}$ Meanwhile, Kaplan et al recently reported that both Baerveldt implant and trabeculectomy with mitomycin $\mathrm{C}$ are more cost-effective than maximal medical treatment. ${ }^{58}$ While there are no data on cost-effectiveness of MIGS yet, if long-term efficacy of MIGS is demonstrated in future clinical studies, MIGS may also prove more cost-effective than medical treatment.

Nonetheless, there are several limitations to the current state of MIGS. These include limited quality and duration of evidence, lack of study standardization, lack of cost-effectiveness data, and incomplete knowledge of ideal patient selection.

MIGS evidence is currently limited by the retrospective and non-masked nature in the majority of cases. Directly comparing the evidence of each MIGS type is difficult due to the varied study designs, patient populations, and outcome measures. Long-term outcomes over several years are mostly unknown. In assessing current MIGS data, because most trials have included cataract surgery, it is important for clinicians to recognize the IOP-lowering ability of cataract surgery alone. ${ }^{59-61}$

According to a recent review by the American Academy of Ophthalmology, cataract surgery results in a small, moderate, and marked reduction in IOP and medications for POAG, PXG, and primary angle-closure glaucoma, respectively. ${ }^{61}$ In studies where MIGS surgery has only been reported in combination with cataract surgery, clinicians cannot assume that IOP-lowering abilities will be similar when cataract surgery is not also performed. Additionally, nearly all of the current MIGS procedures have the potential risk of late failure due to scarring, ${ }^{62}$ and longer follow-up periods in future studies will be needed to tell us how the longevity of these MIGS procedures compares to the less than ideal longevity of SLT.

The FDA has drafted recommendations, still under review, for future MIGS clinical trials that clearly specify the standards that future studies should uphold. ${ }^{63}$ These include: specific visual-field and nerve criteria for patients with mildto-moderate glaucoma; glaucoma stable enough to undergo a medication washout period both at baseline and follow-up for purer IOP outcome measurements; plotting diurnal IOP measurements and using mean diurnal IOPs rather than single daily measurements; a 12-month follow-up requirement; selection criteria that avoid regression to the mean bias; and a recommended primary effectiveness endpoint that will be the percentage of participants with an IOP reduction of $\geq 20 \%$. When future MIGS trials follow more standardized study designs, we will gain an improved understanding of the true IOP-reducing capabilities of each MIGS procedure. Also, once more data from randomized controlled trials are available on each MIGS procedure, subsequent cost-effectiveness analyses will help us to understand the long-term economic benefits of these procedures.

While the current MIGS procedures are generally designed to treat patients with mild-to-moderate OAG, clinicians will need to learn which specific patients will or will not benefit from a particular MIGS procedure. The specific clinical indications that have been learned to date were discussed under the "Adverse events and clinical considerations" sections for each procedure. In general, the trabecular procedures will not benefit patients with elevated episcleral venous pressure. Patients with a bleeding predisposition are less ideal for GATT and possibly for Trabectome as well. It is also interesting to note that in the trabecular procedures, patients with higher baseline IOPs seemed to demonstrate the greatest IOP-lowering effects. These data are not yet available for the non-trabecular procedures.

Future data will help clinicians to individualize their management strategy for each patient. Advances in aqueous angiography imaging will allow clinicians to localize the most active collector channels preoperatively, before deciding where to place a particular trabecular stent. ${ }^{64}$ Such imaging modalities may also assess the activity of uveoscleral flow, thus informing placement location for uveoscleral stents. Perhaps in the future, these diagnostic studies will determine which class of MIGS procedure would be most efficacious for a particular patient. Because future trials will follow more standardized clinical trial protocols, our ability to select appropriate patients for each MIGS type will become more optimized. Future-generation MIGS devices will aim to surpass current MIGS outcomes, and these devices have the ever-increasing potential to improve the lives of patients with glaucoma worldwide.

\section{Disclosure}

Grace M Richter was a Heed Fellow and the David Mays II Glaucoma Fellow at Jules Stein Eye Institute, UCLA. Anne L Coleman is a Reichert consultant and has a First 5 LA Grant. The authors report no other conflicts of interest in this work. 


\section{References}

1. Tham YC, Li X, Wong TY, Quigley HA, Aung T, Cheng CY. Global prevalence of glaucoma and projections of glaucoma burden through 2040: a systematic review and meta-analysis. Ophthalmology. 2014; 121:2081-2090.

2. Heijl A, Leske MC, Bengtsson B, Hyman L, Bengtsson B, Hussein M; Early Manifest Glaucoma Trial Group. Reduction of intraocular pressure and glaucoma progression: results from the Early Manifest Glaucoma Trial. Arch Ophthalmol. 2002;120:1268-1279.

3. Lemij HG, Hoevenaars JG, van der Windt C, Baudouin C. Patient satisfaction with glaucoma therapy: reality or myth? Clin Ophthalmol. 2015;9:785-793.

4. Leahy KE, White AJ. Selective laser trabeculoplasty: current perspectives. Clin Ophthalmol. 2015;9:833-841.

5. Woo DM, Healey PR, Graham SL, Goldberg I. Intraocular pressurelowering medications and long-term outcomes of selective laser trabeculoplasty. Clin Experiment Ophthalmol. 2015;43:320-327.

6. Gedde SJ, Herndon LW, Brandt JD, Budenz DL, Feuer WJ, Schiffman JC; Tube Versus Trabeculectomy Study Group. Postoperative complications in the Tube Versus Trabeculectomy (TVT) study during five years of follow-up. Am J Ophthalmol. 2012;153:804-814.

7. Gedde SJ, Schiffman JC, Feuer WJ, Herndon LW, Brandt JD, Budenz DL; Tube versus Trabeculectomy Study Group. Treatment outcomes in the Tube Versus Trabeculectomy (TVT) study after five years of follow-up. Am J Ophthalmol. 2012;153:789-803.

8. Johnson M. 'What controls aqueous humour outflow resistance?'. Exp Eye Res. 2006;82:545-557.

9. Minckler DS, Baerveldt G, Alfaro MR, Francis BA. Clinical results with the Trabectome for treatment of open-angle glaucoma. Ophthalmology. 2005;112:962-967.

10. Minckler D, Mosaed S, Dustin L, Ms BF; Trabectome Study Group. Trabectome (trabeculectomy-internal approach): additional experience and extended follow-up. Trans Am Ophthalmol Soc. 2008;106: 149-160.

11. Francis BA, See RF, Rao NA, Minckler DS, Baerveldt G. Ab interno trabeculectomy: development of a novel device (Trabectome) and surgery for open-angle glaucoma. J Glaucoma. 2006;15:68-73.

12. Minckler D, Baerveldt G, Ramirez MA, et al. Clinical results with the Trabectome, a novel surgical device for treatment of open-angle glaucoma. Trans Am Ophthalmol Soc. 2006;104:40-50.

13. Maeda M, Watanabe M, Ichikawa K. Evaluation of trabectome in open angle glaucoma. J Glaucoma. 2013;22:205-208.

14. Francis BA, Minckler D, Dustin L, et al; Trabectome Study Group. Combined cataract extraction and trabeculotomy by the internal approach for coexisting cataract and open-angle glaucoma: initial results. J Cataract Refract Surg. 2008;34:1096-1103.

15. Ting JL, Damji KF, Stiles MC; Trabectome Study Group. Ab interno trabeculectomy: outcomes in exfoliation versus primary open-angle glaucoma. J Cataract Refract Surg. 2012;38:315-323.

16. Damji KF, Konstas AG, Liebmann JM, et al. Intraocular pressure following phacoemulsification in patients with and without exfoliation syndrome: a 2 year prospective study. Br J Ophthalmol. 2006;90: 1014-1018.

17. Ahuja Y, Ma Khin Pyi S, Malihi M, Hodge DO, Sit AJ. Clinical results of ab interno trabeculotomy using the trabectome for open-angle glaucoma: the Mayo clinic series in Rochester, Minnesota. Am JOphthalmol. 2013;156:927-335.

18. Jordan JF, Wecker T, van Oterendorp C, et al. Trabectome surgery for primary and secondary open angle glaucomas. Graefes Arch Clin Exp Ophthalmol. 2013;251:2753-2760.

19. Ahuja Y, Malihi M, Sit AJ. Delayed-onset symptomatic hyphema after ab interno trabeculotomy surgery. Am J Ophthalmol. 2012;154: 476-480.

20. Luebke J, Boehringer D, Neuburger M, et al. Refractive and visual outcomes after combined cataract and trabectome surgery: a report on the possible influences of combining cataract and trabectome surgery on refractive and visual outcomes. Graefes Arch Clin Exp Ophthalmol. 2015;253:419-423.
21. Klamann MK, Gonnermann J, Maier AK, Bertelmann E, Joussen AM, Torun N. Influence of selective laser trabeculoplasty (SLT) on combined clear cornea phacoemulsification and Trabectome outcomes. Graefes Arch Clin Exp Ophthalmol. 2014;252:627-631.

22. Voskanyan L, García-Feijoó J, Belda JI, Fea A, Jünemann A, Baudouin C; Synergy Study Group. Prospective, unmasked evaluation of the iStent ${ }^{\circledR}$ inject system for open-angle glaucoma: synergy trial. Adv Ther. 2014;31:189-201.

23. Hunter KS, Fjield T, Heitzmann H, Shandas R, Kahook MY. Characterization of micro-invasive trabecular bypass stents by ex vivo perfusion and computational flow modeling. Clin Ophthalmol. 2014;8:499-506

24. Bahler CK, Hann CR, Fjield T, Haffner D, Heitzmann H, Fautsch MP. Second-generation trabecular meshwork bypass stent (iStent inject) increases outflow facility in cultured human anterior segments. Am J Ophthalmol. 2012;153(6):1206-1213.

25. Fea AM. Phacoemulsification versus phacoemulsification with micro-bypass stent implantation in primary open-angle glaucoma: randomized double-masked clinical trial. J Cataract Refract Surg. 2010;36:407-412.

26. Samuelson TW, Katz LF, Wells JM, Duh YJ, Giamporcaro JE; US iStent Study Group. Randomized evaluation of the trabecular micro-bypass stent with phacoemulsification in patients with glaucoma and cataract. Ophthalmology. 2011;118:459-467.

27. Craven ER, Katz LJ, Wells JM, Giamporcaro JE; iStent Study Group. Cataract surgery with trabecular micro-bypass stent implantation in patients with mild-to-moderate open-angle glaucoma and cataract: two-year follow-up. J Cataract Refract Surg. 2012;38:1339-1345.

28. Fernández-Barrientos Y, García-Feijoó J, Martínez-de-la-Casa JM, Pablo LE, Fernández-Pérez C, García Sánchez J. Fluorophotometric study of the effect of the glaukos trabecular microbypass stent on aqueous humor dynamics. Invest Ophthalmol Vis Sci. 2010;51: 3327-3332.

29. Belovay GW, Naqi A, Chan BJ, Rateb M, Ahmed II. Using multiple trabecular micro-bypass stents in cataract patients to treat open-angle glaucoma. J Cataract Refract Surg. 2012;38:1911-1917.

30. Fea AM, Belda JI, Rekas M, et al. Prospective unmasked randomized evaluation of the iStent inject $\left({ }^{\circledR}\right)$ versus two ocular hypotensive agents in patients with primary open-angle glaucoma. Clin Ophthalmol. 2014; 8:875-882.

31. Klamann M, Gonnermann J, Pahlitzsch M, et al. iStent inject in phakic open angle glaucoma. Graefes Arch Clin Exp Ophthalmol. 2015; 253:941-947.

32. Johnstone MA, Grant WG. Pressure-dependent changes in structures of the aqueous outflow system of human and monkey eyes. Am J Ophthalmol. 1973;75:365-383.

33. Pfeiffer N, Garcia-Feijoo J, Martinez-de-la-Casa JM, et al. A randomized trial of a Schlemm's canal microstent with phacoemulsification for reducing intraocular pressure in open-angle glaucoma. Ophthalmology. 2015;122:1283-1293.

34. Grover DS, Godfrey DG, Smith O, Feuer WJ, Montes de Oca I, Fellman RL. Gonioscopy-assisted transluminal trabeculotomy, ab interno trabeculotomy: technique report and preliminary results. Ophthalmology. 2014;121:855-861.

35. Beck AD, Lynch MG. 360 degrees trabeculotomy for primary congenital glaucoma. Arch Ophthalmol. 1995;113:1200-1202.

36. Chin S, Nitta T, Shinmei Y, et al. Reduction of intraocular pressure using a modified 360-degree suture trabeculotomy technique in primary and secondary open-angle glaucoma: a pilot study. J Glaucoma. 2012; 21:401-407.

37. Grover DS, Smith O, Fellman RL, et al. Gonioscopy assisted transluminal trabeculotomy: an ab interno circumferential trabeculotomy for the treatment of primary congenital glaucoma and juvenile open angle glaucoma. Br J Ophthalmol. 2015;99(8):1092-1096.

38. Berlin MS, Rajacich G, Duffy M, Grundfest W, Goldenberg T. Excimer laser photoablation in glaucoma filtering surgery. Am J Ophthalmol. 1987; 103:713-714. 
39. Babighian S, Caretti L, Tavolato M, Cian R, Galan A. Excimer laser trabeculotomy vs 180 degrees selective laser trabeculoplasty in primary open-angle glaucoma. A 2-year randomized, controlled trial. Eye (Lond). 2010;24:632-638.

40. Töteberg-Harms M, Hanson JV, Funk J. Cataract surgery combined with excimer laser trabeculotomy to lower intraocular pressure: effectiveness dependent on preoperative IOP. BMC Ophthalmol. 2013;13:24.

41. Toris CB, Yablonski ME, Wang YL, Camras CB. Aqueous humor dynamics in the aging human eye. Am J Ophthalmol. 1999;127:407-412.

42. Emi K, Pederson JE, Toris CB. Hydrostatic pressure of the suprachoroidal space. Invest Ophthalmol Vis Sci. 1989;30:233-238.

43. Saheb H, Ianchulev T, Ahmed II. Optical coherence tomography of the suprachoroid after CyPass Micro-Stent implantation for the treatment of open-angle glaucoma. Br J Ophthalmol. 2014;98:19-23.

44. Hoeh H, Ahmed IK, Grisanti S, et al. Early postoperative safety and surgical outcomes after implantation of a suprachoroidal micro-stent for the treatment of open-angle glaucoma concomitant with cataract surgery. J Cataract Refract Surg. 2013;39:431-437.

45. García-Feijoo J, Rau M, Grisanti S, et al. Supraciliary micro-stent implantation for open-angle glaucoma failing topical therapy: 1-year results of a multicenter study. Am J Ophthalmol. 2015;159:1075-1081.

46. Francis BA, Berke SJ, Dustin L, Noecker R. Endoscopic cyclophotocoagulation combined with phacoemulsification versus phacoemulsification alone in medically controlled glaucoma. J Cataract Refract Surg. 2014;40:1313-1321.

47. Siegel MJ, Boling WS, Faridi OS, et al. Combined endoscopic cyclophotocoagulation and phacoemulsification versus phacoemulsification alone in the treatment of mild to moderate glaucoma. Clin Experiment Ophthalmol. 2015;43:531-539.

48. Tan JC, Francis BA, Noecker R, Uram M, Dustin L, Chopra V. Endoscopic cyclophotocoagulation and pars plana ablation (ECP-Plus) to treat refractory glaucoma. J Glaucoma. Epub 2015 May 14.

49. Francis BA, Pouw A, Jenkins D, et al. Endoscopic cycloplasty (ECPL) and lens extraction in the treatment of severe plateau iris syndrome. J Glaucoma. Epub 2015 Mar 18.

50. Lewis RA. Ab interno approach to the subconjunctival space using a collagen glaucoma stent. J Cataract Refract Surg. 2014;40:1301-1306.

51. Tsai JC. Medication adherence in glaucoma: approaches for optimizing patient compliance. Curr Opin Ophthalmol. 2006;17:190-195.

52. Dreer LE, Girkin CA, Campbell L, Wood A, Gao L, Owsley C. Glaucoma medication adherence among African Americans: program development. Optom Vis Sci. 2013;90:883-897.

53. Lacey J, Cate H, Broadway DC. Barriers to adherence with glaucoma medications: a qualitative research study. Eye (Lond). 2009;23: 924-932.
54. Friedman DS, Hahn SR, Gelb L, et al. Doctor-patient communication, health-related beliefs, and adherence in glaucoma results from the Glaucoma Adherence and Persistency Study. Ophthalmology. 2008;115:1320-1327.

55. Kaštelan S, Tomić M, Metež Soldo K, Salopek-Rabatić J. How ocular surface disease impacts the glaucoma treatment outcome. Biomed Res Int. 2013;2013:696328.

56. Baudouin C, Renard JP, Nordmann JP, et al. Prevalence and risk factors for ocular surface disease among patients treated over the long term for glaucoma or ocular hypertension. Eur J Ophthalmol. Epub 2012 Jun 11.

57. Stein JD, Kim DD, Peck WW, Giannetti SM, Hutton DW. Costeffectiveness of medications compared with laser trabeculoplasty in patients with newly diagnosed open-angle glaucoma. Arch Ophthalmol. 2012;130:497-505

58. Kaplan RI, De Moraes CG, Cioffi GA, Al-Aswad LA, Blumberg DM. Comparative cost-effectiveness of the Baerveldt implant, trabeculectomy with mitomycin, and medical treatment. JAMA Ophthalmol. 2015;133:560-567.

59. Yang HS, Lee J, Choi S. Ocular biometric parameters associated with intraocular pressure reduction after cataract surgery in normal eyes. Am J Ophthalmol. 2013;156:89-94.

60. Mansberger SL, Gordon MO, Jampel H, et al; Ocular Hypertension Treatment Study Group. Reduction in intraocular pressure after cataract extraction: the Ocular Hypertension Treatment Study. Ophthalmology. 2012;119:1826-1831

61. Chen PP, Lin SC, Junk AK, Radhakrishnan S, Singh K, Chen TC. The effect of phacoemulsification on intraocular pressure in glaucoma patients: a report by the American Academy of Ophthalmology. Ophthalmology. 2015;122:1294-1307.

62. Schmidt W, Kastner C, Sternberg K, et al. New concepts for glaucoma implants - controlled aqueous humor drainage, encapsulation prevention and local drug delivery. Curr Pharm Biotechnol. 2013;14:98-111.

63. Premarket Studies of Implantable Minimally Invasive Glaucoma Surgical (MIGS) Devices: Draft Guidance for Industry and Food and Drug Administration Staff. Rockville: Food and Drug Administration; 2015. Available from: http://www.fda.gov/downloads/MedicalDevices/ DeviceRegulationandGuidance/GuidanceDocuments/UCM433165.pdf. Accessed July 1, 2015.

64. van der Merwe EL, Kidson SH. Advances in imaging the blood and aqueous vessels of the ocular limbus. Exp Eye Res. 2010;91:118-126.
Clinical Ophthalmology

\section{Publish your work in this journal}

Clinical Ophthalmology is an international, peer-reviewed journal covering all subspecialties within ophthalmology. Key topics include: Optometry; Visual science; Pharmacology and drug therapy in eye diseases; Basic Sciences; Primary and Secondary eye care; Patient Safety and Quality of Care Improvements. This journal is indexed on Submit your manuscript here: http://www.dovepress.com/clinical-ophthalmology-journal
Dovepress

PubMed Central and CAS, and is the official journal of The Society of Clinical Ophthalmology (SCO). The manuscript management system is completely online and includes a very quick and fair peer-review system, which is all easy to use. Visit http://www.dovepress.com/ testimonials.php to read real quotes from published authors. 